\title{
A NON ELLIPTIC SPECTRAL PROBLEM RELATED TO THE ANALYSIS OF SUPERCONDUCTING MICRO-STRIP LINES
}

\author{
Anne-Sophie Bonnet-BendhiA ${ }^{1}$ And KARIM RAMdAni ${ }^{2}$
}

\begin{abstract}
This paper is devoted to the spectral analysis of a non elliptic operator $A$, deriving from the study of superconducting micro-strip lines. Once a sufficient condition for the self-adjointness of operator $A$ has been derived, we determine its continuous spectrum. Then, we show that $A$ is unbounded from below and that it has a sequence of negative eigenvalues tending to $-\infty$. Using the Min-Max principle, a characterization of its positive eigenvalues is given. Thanks to this characterization, some conditions on the geometrical (large width) and physical (large dielectric permittivity in modulus) properties of the strip that ensure the existence of positive eigenvalues are derived. Finally, we analyze the asymptotic behavior of the eigenvalues of $A$ as the dielectric permittivity of the strip goes to $-\infty$.
\end{abstract}

Mathematics Subject Classification. 35P, 47A70, 47B25, 78A50.

Received: October 11, 2001. Revised: February 25, 2002.

\section{INTRODUCTION}

The micro-strip transmission line is one of the most commonly used lines in micro-wave integrated circuits, in order to connect to each other the different micro-electronic components $(c f .[7,12])$. It is constituted of a thin conducting strip located on a dielectric substrate, which is placed on a conducting ground plane. The line behaves then as an electromagnetic wave-guide, since the energy of the guided modes is confined in the dielectric area located between the conducting strip and the ground plane. Since the discovery of high- $T_{c}$ superconducting materials fifteen years ago, new lines involving superconducting strips have been realized in order to reduce thermic losses.

As usual in the open wave-guide theory $[1,2,4,8]$, the determination of the guided modes of the superconducting micro-strip line amounts to solve an eigenvalue problem associated to a non compact operator. Because of this non-compactness property, the existence of eigenvalues (i.e. the existence of a discrete spectrum), and thus the existence of guided modes, is not ensured. To answer this question, the procedure classically followed in the literature is first to show the self-adjointness of the operator involved in the spectral problem, then to determine its continuous spectrum, and finally to use the Min-Max principle to study the existence of eigenvalues. In the problem studied in this paper, these steps present several specific difficulties, all of them induced by the superconducting properties of the strip. Indeed, in the framework of London's model, superconducting materials

\footnotetext{
Keywords and phrases. Superconducting transmission lines, wave-guides, self-adjointness, spectral analysis, non elliptic operators.

${ }^{1}$ Laboratoire SMP, ENSTA, URA 853 du CNRS, 32 Boulevard Victor, 75739 Paris Cedex 15, France. e-mail: bonnet@ensta.fr

2 Projet CORIDA, INRIA Lorraine, Campus Scientifique, BP 239, 54506 Vandœuvre-les-Nancy, France.

e-mail: ramdani@loria.fr
} 
behave as negative dielectric permittivity materials $(c f .[9,11])$. The change of sign of the permittivity in the line (negative inside the strip and positive outside) destroys the ellipticity properties of the operator $A$ involved in our spectral problem. The originality of the present work lies in the treatment of this lack of ellipticity and its consequences on the spectral analysis of $A$ (self-adjointness, continuous spectrum, characterization of positive eigenvalues). In [5], an elliptic vector model corresponding to a zero thickness strip (i.e. a crack) has been studied. The superconducting properties of the strip were there taken into account through an impedance condition written on its boundary. In this paper, we investigate the difficulties induced by the negative value of the dielectric permittivity of the strip, in the framework of a non elliptic scalar model. Let us emphasize here that this problem cannot be considered as an approximation of the physical vector one. However, it gives rise to similar mathematical difficulties and that's why its study is a necessary preliminary step.

The paper is organized as follows: Section 1 is devoted to the description of the studied scalar model. In Section 2, we give a sufficient condition on the dielectric permittivity of the strip $\varepsilon_{S}$ for self-adjointness. This result is obtained thanks to a perturbation method, using the Dirichlet to Neumann operator. Let us emphasize here that in [3], more precise results about self-adjointness in non elliptic transmission problems have been obtained in the paticular case (and simpler compared to the one studied here) where the exterior of the strip is supposed to be homogeneous (see Rem. 2.5). Section 3 is devoted to the determination of the essential spectrum. We prove that this continuous spectrum is the same as the one of the operator corresponding to the perfectly stratified medium obtained after removing the strip. In Section 4, we prove that the operator $A$ involved in our model has a sequence of negative eigenvalues tending to $-\infty$. Thus, it is not bounded from below, and the Min-Max principle cannot be applied to it. In Section 5, a method to overcome this difficulty is proposed. This method is based on the application of the Min-Max principle not to $A$, but to its inverse $A^{-1}$. One of the advantages of this method is that it allows one to characterize the positive eigenvalues, which are the only interesting ones from the physical viewpoint. Using the characterization derived, we give then sufficient conditions on the width and the dielectric permittivity of the strip ensuring the existence of a given number of positive eigenvalues. This is achieved using the properties of the one-dimensional problem corresponding to the case of an infinitely wide strip. Finally, in Section 6, it is shown that the more standard case (since it is elliptic) of a perfectly conducting strip can be seen as the asymptotic limit of our problem when the permittivity of the strip goes to $-\infty$. This asymptotic analysis requires the study of a singular perturbation problem, involving a skin effect. The results obtained are then used to derive an asymptotic existence result for positive eigenvalues.

\section{Mathematical Setting of the Problem}

Let us start by introducing the notations used throughout the paper.

Given an orthonormal system of coordinates $\left(O, x_{1}, x_{2}, x_{3}\right)$, let the micro-strip line occupy the half-space $x_{2}>0$, and suppose that the direction of propagation is $x_{3}$. We denote by $x=\left(x_{1}, x_{2}\right)$ the current point of the cross section of the line $\Omega=\left\{x ; x_{2}>0\right\}$.

The superconducting strip, the dielectric substrate and the air are supposed to occupy respectively the domains having the following sections (see Fig. 1):

- $\Omega_{S}=\left\{x=\left(x_{1}, x_{2}\right),\left|x_{1}\right|<\frac{\ell}{2}, h<x_{2}<h+d\right\}$, with boundary $\partial \Omega_{S}=\Gamma$.

- $\Omega_{D}=\left\{x=\left(x_{1}, x_{2}\right), 0<x_{2}<h\right\}$, with boundary $\partial \Omega_{D}=\Gamma_{P} \cap \Sigma$ where $\Gamma_{P}=\left\{x_{2}=0\right\}$ denotes the cross section of the perfectly conducting ground plane, and $\Sigma=\left\{x_{2}=h\right\}$ the one of the interface between the dielectric and the air.

- $\Omega_{A}=\Omega \backslash \overline{\left(\Omega_{D} \cup \Omega_{S}\right)}$.

As it has been already said, superconducting materials behave in the framework of London's model for superconductivity $(c f .[9,11])$ as materials having negative dielectric permittivity. We shall thus assume in the sequel 


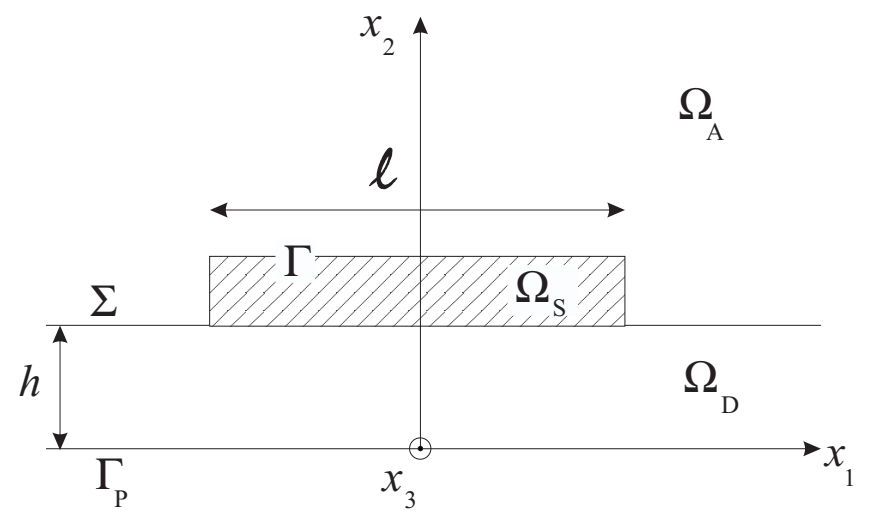

FiguRE 1. The cross section of the micro-strip line.

that the relative dielectric permittivity at any point $x=\left(x_{1}, x_{2}\right)$ of the cross section $\Omega$ is defined as follows:

$$
\varepsilon(x)= \begin{cases}\varepsilon_{D}>1, & x \in \Omega_{D} \\ \varepsilon_{S}<0, & x \in \Omega_{S} \\ \varepsilon_{A}=1, & x \in \Omega_{A} .\end{cases}
$$

In the model studied in this paper, the guided modes we are looking for are functions of the form:

$$
\left\{\begin{array}{c}
\mathbf{u}\left(x, x_{3}, t\right)=u(x) \mathrm{e}^{i\left(\omega t-\beta x_{3}\right)}, \\
u \in L^{2}(\Omega) \quad \text { and } \quad \omega, \beta \in \mathbb{R}^{*}
\end{array}\right.
$$

satisfying:

$$
\left\{\begin{aligned}
\frac{\partial^{2} \mathbf{u}}{\partial t^{2}}-\operatorname{div}\left(\frac{1}{\varepsilon} \nabla \mathbf{u}\right) & =0 & & (\Omega \times \mathbb{R}) \\
\partial_{n} \mathbf{u} & =0 & & \left(\Gamma_{P} \times \mathbb{R}\right)
\end{aligned}\right.
$$

where $\partial_{n}$ denotes the normal derivative.

\section{Remark 1.1.}

(i) The function $\mathbf{u}$ defined by (1) describes a wave propagating in the $x_{3}$-direction without attenuation nor deformation, with the velocity $\omega / \beta$.

(ii) The operator involved in problem (2) is the one that appears in the description of the transverse magnetic modes of the micro-strip line, in the case of a strip of infinite width.

(iii) The boundary condition on $\Gamma_{P} \times \mathbb{R}$ is imposed to take into account the fact that the ground plane is perfectly conducting.

(iv) In fact, in the complete London's model for superconductivity, the dielectric permittivity of a superconducting material is not only negative but also frequency-dependent. More precisely, we have:

$$
\varepsilon_{S}=\varepsilon_{S}(\omega)=-1 /\left(\omega^{2} \lambda_{L}^{2}\right)
$$

where $\lambda_{L}$ is called the London's length and represents the penetration depth of the electromagnetic field inside the superconducting material. For the sake of simplicity, this dependence of $\varepsilon_{S}$ upon frequency has been omitted in the model studied here. Nevertheless, the analysis of the complete model (which is non linear with respect 
to the frequency $\omega$ ) can be carried out by coupling the arguments developped in this paper to a fixed point method (cf. [13]).

Substituting (1) into (2) leads immediately to the following eigenvalue problem for the function $u$ describing the transverse behavior of the guided mode $\mathbf{u}$ :

$$
\left\{\begin{aligned}
-\operatorname{div}\left(\frac{1}{\varepsilon} \nabla u\right)+\frac{\beta^{2}}{\varepsilon} u & =\omega^{2} u \quad(\Omega) \\
\partial_{n} u & =0 \quad\left(\Gamma_{P}\right) .
\end{aligned}\right.
$$

In other words, if $\beta \neq 0$ is considered as a parameter, a guided mode can be defined as a solution $\left(\omega^{2}, u\right)$ of $(3)$ satisfying $\omega^{2} \neq 0$ and $u \neq 0$.

Let us now define a convenient functional framework to study problem (3).

Introduce the unbounded operator $A$ of $L^{2}(\Omega)$, with domain:

$$
D(A)=\left\{u \in H^{1}(\Omega), \operatorname{div}\left(\frac{1}{\varepsilon} \nabla u\right) \in L^{2}(\Omega), \partial_{n} u_{\mid \Gamma_{P}}=0\right\},
$$

and such that for $u \in D(A)$ :

$$
A u=-\operatorname{div}\left(\frac{1}{\varepsilon} \nabla u\right)+\frac{\beta^{2}}{\varepsilon} u .
$$

The guided modes we are looking for are then solutions of the spectral problem:

$$
\left\{\begin{array}{c}
\text { For } \beta>0, \text { find }(\omega, u) \in \mathbb{R}^{*} \times D(A), u \neq 0, \text { such that: } \\
A u=\omega^{2} u
\end{array}\right.
$$

Throughout all this paper, $L^{2}(\Omega)$ will be equipped with the scalar product:

$$
(u, v)=\int_{\Omega} u \bar{v}
$$

and the associated norm will be noted:

$$
\|u\|_{L^{2}}=\left(\int_{\Omega}|u|^{2}\right)^{1 / 2}
$$

With this hilbertian structure, $A$ is the unbounded operator of $L^{2}(\Omega)$ associated to the bilinear form $a(\cdot, \cdot)$ defined by:

$$
\left\{\begin{array}{l}
D(a)=H^{1}(\Omega) \\
a(u, v)=\int_{\Omega} \frac{1}{\varepsilon}\left(\nabla u \cdot \nabla \bar{v}+\beta^{2} u \bar{v}\right) .
\end{array}\right.
$$

To carry out the spectral analysis of $A$, we shall start by studying its self-adjointness.

\section{About the Self-Adjointness of $A$}

Operator $A$ is clearly symmetric since it is associated to the symmetric bilinear form $a(\cdot, \cdot)$ defined by $(5)$. We know from classical characterizations of self-adjoint operators that a sufficient condition for self-adjointness of symmetric operators is their surjectivity. Here, because of the change of sign of the permittivity $\varepsilon$, the bilinear 
form $a(\cdot, \cdot)$ is not coercive, and the surjectivity of $A$ is far from being obvious. The method we propose to answer this question is based on a perturbation result. More precisely, we shall prove that for "small" or "large" values of the dielectric permittivity $\varepsilon_{S}$ inside the strip, operator $A$ is self-adjoint.

Before stating this result, let us introduce some further notations needed here. We will denote by $\Omega_{E}=\Omega \backslash \overline{\Omega_{S}}$ the cross section of the exterior domain, i.e. the domain located outside the strip. The outgoing unitary normal vector to $\Omega_{S}$ is noted $n$. Throughout this section, $v_{S}$ (respectively $v_{E}$ ) will denote for a given function $v$ defined on $\Omega$ its restriction to $\Omega_{S}$ (respectively $\Omega_{E}$ ). In particular, the function defining the dielectric permittivity in the cross section of the line satisfies thus:

$$
\varepsilon(x)= \begin{cases}\varepsilon_{S}<0 & \text { for } x \in \Omega_{S} \\ \varepsilon_{E}(x)>0 & \text { for } x \in \Omega_{E}\end{cases}
$$

where $\varepsilon_{E}(x)=\varepsilon_{A}=1$ in $\Omega_{A}$ and $\varepsilon_{E}(x)=\varepsilon_{D}>1$ in $\Omega_{D}$.

To study the self-adjointness of $A$, we are going to study the existence of solutions for the equation:

$$
A u=f
$$

where $f$ is a given function in $L^{2}(\Omega)$.

To achieve this, the idea is to transform problem (6) into an equivalent one whose unknown will be the trace $\varphi=u_{\mid \Gamma} \in H^{1 / 2}(\Gamma)$. As a first step, one should notice that problem (6) can be written equivalently as a transmission problem through the interface $\Gamma$, with unknowns $u=\left(u_{S}, u_{E}\right) \in H^{1}\left(\Omega_{S}\right) \times H^{1}\left(\Omega_{E}\right)$ :

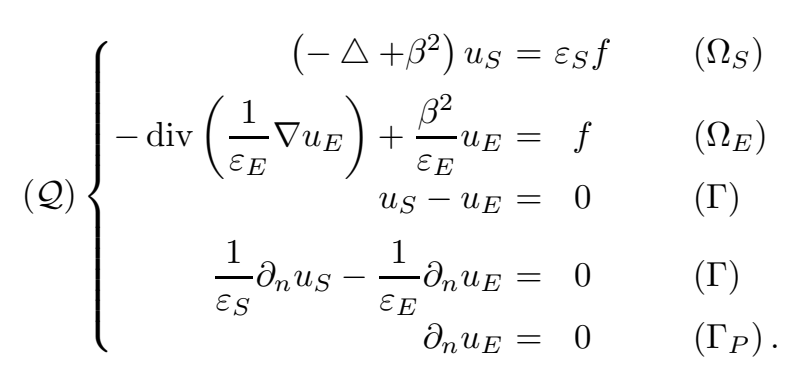

Remark 2.1. Since $u_{E} \in H^{1}\left(\Omega_{E}\right)$ satisfies $\operatorname{div}\left(\frac{1}{\varepsilon_{E}} \nabla u_{E}\right) \in L^{2}\left(\Omega_{E}\right)$, its normal derivative $\frac{1}{\varepsilon_{E}} \partial_{n} u_{E \mid \Gamma}$ can be defined in $H^{-1 / 2}(\Gamma)$ thanks to the following Green formula that holds for any $v_{E} \in H^{1}\left(\Omega_{E}\right)$ :

$$
\left\langle\frac{1}{\varepsilon_{E}} \partial_{n} u_{E \mid \Gamma}, v_{E \mid \Gamma}\right\rangle_{\Gamma}=-\int_{\Omega_{E}} \operatorname{div}\left(\frac{1}{\varepsilon_{E}} \nabla u_{E}\right) \overline{v_{E}} \mathrm{~d} x-\int_{\Omega_{E}} \frac{1}{\varepsilon_{E}} \nabla u_{E} \cdot \nabla \overline{v_{E}} \mathrm{~d} x,
$$

the brackets $\langle\cdot, \cdot\rangle_{\Gamma}$ denoting here the duality between $H^{-1 / 2}(\Gamma)$ and $H^{1 / 2}(\Gamma)$.

As a second step, we transform the transmission problem $(\mathcal{Q})$ into an equivalent one, noted $(\mathcal{P})$, in which the only (non-zero) data appears in the normal derivative transmission condition. To achieve this, we use the well-posedness of the following elliptic (since $\beta \neq 0$ ) Dirichlet problems respectively in $H^{1}\left(\Omega_{S}\right)$ and $H^{1}\left(\Omega_{E}\right)$ :

$$
\left\{\begin{array} { r l } 
{ ( - \triangle + \beta ^ { 2 } ) w _ { S } = \varepsilon _ { S } f } & { ( \Omega _ { S } ) } \\
{ w _ { S } = 0 } & { ( \Gamma ) }
\end{array} \quad \left\{\begin{array}{rl}
-\operatorname{div}\left(\frac{1}{\varepsilon_{E}} \nabla w_{E}\right)+\frac{\beta^{2}}{\varepsilon_{E}} w_{E}=f\left(\Omega_{E}\right) \\
w_{E}=0(\Gamma) \\
\partial_{n} w_{E}=0\left(\Gamma_{P}\right) .
\end{array}\right.\right.
$$


Consequently, the couple $\left(u_{S}, u_{E}\right)$ solves $(\mathcal{Q})$ if and only if $\left(v_{S}, v_{E}\right)=\left(u_{S}-w_{S}, u_{E}-w_{E}\right)$ satisfies:

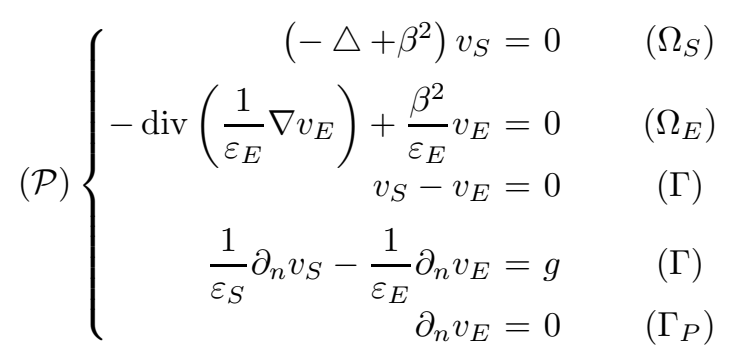

where we have set:

$$
g=-\left(\frac{1}{\varepsilon_{S}} \partial_{n} w_{S}-\frac{1}{\varepsilon_{E}} \partial_{n} w_{E}\right)_{\mid \Gamma} \in H^{-1 / 2}(\Gamma) .
$$

Now, to study the well-posedness of $(\mathcal{P})$, we introduce the Dirichlet to Neumann operators $T_{S}$ and $T_{E}$, defined for any trace $\varphi$ in $H^{1 / 2}(\Gamma)$ by the relations:

$$
\left\{\begin{array}{l}
T_{S} \varphi=\partial_{n} v_{S}(\varphi)_{\mid \Gamma} \\
T_{E} \varphi=\frac{1}{\varepsilon_{S}} \partial_{n} v_{E}(\varphi)_{\mid \Gamma}
\end{array}\right.
$$

where $v_{S}(\varphi)$ and $v_{E}(\varphi)$ solve respectively the interior and exterior Dirichlet problems with data $\varphi$ :

$$
\left\{\begin{array} { r l } 
{ ( - \triangle + \beta ^ { 2 } ) v _ { S } ( \varphi ) } & { = 0 ( \Omega _ { S } ) } \\
{ v _ { S } ( \varphi ) } & { = \varphi ( \Gamma ) }
\end{array} \quad \left\{\begin{array}{rl}
-\operatorname{div}\left(\frac{1}{\varepsilon_{E}} \nabla v_{E}(\varphi)\right)+\frac{\beta^{2}}{\varepsilon_{E}} v_{E}(\varphi)=0 & \left(\Omega_{E}\right) \\
v_{E}(\varphi) & =\varphi \quad(\Gamma) \\
\partial_{n} v_{E}(\varphi) & =0 \quad\left(\Gamma_{P}\right) .
\end{array}\right.\right.
$$

Note that these problems being well-posed, operators $T_{S}$ et $T_{E}$ define isomorphisms from $H^{1 / 2}(\Gamma)$ onto $H^{-1 / 2}(\Gamma)$. Solving $(\mathcal{P})$ is then equivalent to solve the following equation:

$$
\left(\frac{1}{\varepsilon_{S}} T_{S}-T_{E}\right) \varphi=g
$$

We are now able to derive a sufficient condition on $\varepsilon_{S}$ ensuring the well-posedness of $(\mathcal{P})$ :

Lemma 2.2. There exists $c>0$ such that for every $\left.\varepsilon_{S} \in\right]-\infty,-c[\cup]-1 / c, 0[$, problem $(\mathcal{P})$, and thus problem $(\mathcal{Q})$, admits a unique solution.

Proof. Since $T_{E}$ is an isomorphism, the invertibility of $\left(\frac{1}{\varepsilon_{S}} T_{S}-T_{E}\right)$ is equivalent to the invertibility of $\left(\frac{1}{\varepsilon_{S}} T_{E}^{-1} T_{S}-\mathrm{Id}\right)$, which is clearly satisfied if:

$$
\left|\frac{1}{\varepsilon_{S}}\right|\left\|T_{E}^{-1} T_{S}\right\|<1
$$

Consequently, if $\left|\varepsilon_{S}\right|>\left\|T_{E}^{-1} T_{S}\right\|$, problems $(\mathcal{P})$ and $(\mathcal{Q})$ are well-posed. Applying the same kind of arguments to the operator Id $-\varepsilon_{S} T_{S}^{-1} T_{E}$, we can prove the well-posedness of $(\mathcal{P})$ and $(\mathcal{Q})$ for $\left|\varepsilon_{S}\right|<\left\|T_{S}^{-1} T_{E}\right\|^{-1}$. The lemma is thus proved, with: $c=\max \left(\left\|T_{E}^{-1} T_{S}\right\|,\left\|T_{S}^{-1} T_{E}\right\|\right)$. 
Remark 2.3. Notice that the constant $c=\max \left(\left\|T_{E}^{-1} T_{S}\right\|,\left\|T_{S}^{-1} T_{E}\right\|\right)$ satisfies $c \geq 1$, since:

$$
\left\|T_{E}^{-1} T_{S}\right\|\left\|T_{S}^{-1} T_{E}\right\| \geq\left\|T_{E}^{-1} T_{S} T_{S}^{-1} T_{E}\right\|=1
$$

We immediately deduce from this lemma the main result of this section:

Theorem 2.4. There exists a constant $c>0$ such that for:

$$
\left.\varepsilon_{S} \in\right]-\infty,-c[\cup]-1 / c, 0[
$$

the symmetric operator $A$ is invertible and thus self-adjoint.

From now on, we will assume that the dielectric permittivity of the strip $\varepsilon_{S}$ satisfies condition (8), and thus that $A$ is self-adjoint.

\section{Remark 2.5.}

(i) In fact, the result of Theorem 2.4 can be precised in the special case where the exterior of the strip is supposed to be homogeneous (i.e.: $\varepsilon_{D}=\varepsilon_{A}$ ). This particular case, and more generally the question of selfadjointness of non elliptic transmission operators through a regular or polygonal interface between two media has been investigated in [3]. For regular interfaces, it is proved in [3] that the problem is self-adjoint if and only if $\varepsilon_{S} / \varepsilon_{A} \neq-1$. For polygonal interfaces, it is proved that for some negative values of the contrast $\varepsilon_{S} / \varepsilon_{A}$ constituting an interval containing -1 , the operator $A$ is not self-adjoint. More precisely, if the interface has only one corner of angle $0<\theta \leq \pi$, the operator is self-adjoint if and only if

$$
\frac{\varepsilon_{S}}{\varepsilon_{A}} \notin I_{\theta}=\left[\frac{\theta-2 \pi}{\theta}, \frac{\theta}{\theta-2 \pi}\right] .
$$

This result is directly related to the fact that for these values of the contrast, the domain of the adjoint $A^{*}$ of $A$ contains some corner singularities which are too strong $\left(L^{2}\right.$ but not $\left.H^{1}\right)$ to belong to the domain $D(A)$ of $A$.

(ii) In our problem, we can distinguish two kinds of corners: the upper corners of the strip corresponding to a two-media interface, and the lower ones, corresponding to a three-media interface. For the upper ones, the results of [3] can be applied, while for the lower ones, one needs to generalize the method proposed in [3] to the case of a three-media interface. Nevertheless, note that [3] already shows that our operator $A$ is not self-adjoint (at least) for $\varepsilon_{S} \in I_{\pi / 2}=[-3,-1 / 3]$, because of the singularities generated by the upper corners. Consequently, the constant $c$ in Theorem 2.4 satisfies $c>3$.

(iii) In the framework of London's model, the superconducting material becomes better as $\varepsilon_{S}$ decreases, and thus, as the penetration depth of the electromagnetic field inside the strip gets smaller. In particular, the limit case $\varepsilon_{S}=-\infty$ corresponds to a zero penetration depth, and can thus be interpreted as the case of a perfectly conducting strip ( $c f$. Sect. 6).

\section{The essential SPECTRUM}

Since operator $A$ is self-adjoint, its spectrum is real. Moreover, because $\Omega$ is unbounded, the embedding of $D(A)$ in $L^{2}(\Omega)$ is not compact, and thus the spectrum of $A$ has a continuous part, the so-called essential spectrum. Our aim here is to determine this continuous part. Using the fact that the strip has a bounded section, we are going to prove that the essential spectrum of $A$ is identical to the one of the operator $A_{\infty}$ associated to the unperturbed medium. Here, we mean by unperturbed medium the medium constituted by the air and the dielectric subtrate, obtained after the strip has been removed. In other words, the result established here is a stability result for the essential spectrum, under a compact geometric perturbation. The main difficulty of the proof is once again due to the loss of coercivity induced by the change of sign of $\varepsilon$. 
Before stating more precisely the main result of this section, let us introduce some notations in order to define the operator $A_{\infty}$ corresponding to the unperturbed medium. Set:

$$
\varepsilon_{\infty}(x)=\varepsilon_{\infty}\left(x_{2}\right)= \begin{cases}\varepsilon_{D}>1 & \text { in } \Omega_{D} \\ \varepsilon_{A}=1 & \text { in } \Omega \backslash \overline{\Omega_{D}} .\end{cases}
$$

Note that $\varepsilon_{\infty}$ is nothing but the function describing the (relative) dielectric permittivity of the unperturbed medium. Then, define operator $A_{\infty}$ as follows:

$$
\left\{\begin{array}{l}
D\left(A_{\infty}\right)=\left\{u \in H^{1}(\Omega), \operatorname{div}\left(\frac{1}{\varepsilon_{\infty}} \nabla u\right) \in L^{2}(\Omega), \partial_{n} u_{\mid \Gamma_{P}}=0\right\} \\
A_{\infty} u=-\operatorname{div}\left(\frac{1}{\varepsilon_{\infty}} \nabla u\right)+\frac{\beta^{2}}{\varepsilon_{\infty}} u .
\end{array}\right.
$$

The essential spectrum of $A$ is then given by

\section{Theorem 3.1.}

$$
\sigma_{\mathrm{ess}}(A)=\sigma\left(A_{\infty}\right)=\left[\gamma_{\mathrm{TM}},+\infty[\right.
$$

where $\gamma_{T M}$ is defined by the formula:

$$
\gamma_{\mathrm{TM}}=\inf _{u \in H^{1}\left(\mathbb{R}^{+}\right), u \neq 0} \frac{\int_{0}^{+\infty} \frac{1}{\varepsilon_{\infty}\left(x_{2}\right)}\left(\left|\frac{\mathrm{d} u}{\mathrm{~d} x_{2}}\right|^{2}+\beta^{2}|u|^{2}\right) \mathrm{d} x_{2}}{\int_{0}^{+\infty}|u|^{2} \mathrm{~d} x_{2}} .
$$

Remark 3.2. The equation $\omega^{2}=\gamma_{\mathrm{TM}}$ (relating the frequency $\omega$ and the wave-number $\beta$ ) is the dispersion relation of the fundamental transverse magnetic guided mode of the slab waveguide (cf. [10]). From a mathematical point of view, $\gamma_{\mathrm{TM}}$ repesents the first eigenvalue of the self-adjoint operator $A_{\mathrm{TM}}$ defined on $L^{2}\left(\mathbb{R}^{+}\right)$by:

$$
\left\{\begin{array}{l}
D\left(A_{\mathrm{TM}}\right)=\left\{u \in H^{1}\left(\mathbb{R}^{+}\right) ; \frac{\mathrm{d}}{\mathrm{d} x_{2}}\left(\frac{1}{\varepsilon_{\infty}} \frac{\mathrm{d} u}{\mathrm{~d} x_{2}}\right) \in L^{2}\left(\mathbb{R}^{+}\right), \frac{\mathrm{d} u}{\mathrm{~d} x_{2}}(0)=0\right\} \\
A_{\mathrm{TM}} u=-\frac{\mathrm{d}}{\mathrm{d} x_{2}}\left(\frac{1}{\varepsilon_{\infty}} \frac{\mathrm{d} u}{\mathrm{~d} x_{2}}\right)+\frac{\beta^{2}}{\varepsilon_{\infty}} u .
\end{array}\right.
$$

One can easily check (cf. [10]) that $\gamma_{\mathrm{TM}}$ is the first positive solution of the the dispersion relation:

$$
\tan (\kappa h)=\varepsilon_{D} \eta / \kappa
$$

where we have set $\kappa=\sqrt{\gamma_{\mathrm{TM}} \varepsilon_{D}-\beta^{2}}$ and $\eta=\sqrt{\beta^{2}-\gamma_{\mathrm{TM}}}$. Using this property, one can in particular prove that $\gamma_{\mathrm{TM}}$ satisfies:

$$
\frac{\beta^{2}}{\varepsilon_{D}}<\gamma_{\mathrm{TM}}<\beta^{2}
$$

To prove Theorem 3.1, we shall successively prove (in Lems. 3.3, 3.4 and 3.5) the following inclusions:

$$
\sigma\left(A_{\infty}\right) \subset\left[\gamma_{T M},+\infty\left[\quad, \quad\left[\gamma_{\mathrm{TM}},+\infty\left[\subset \sigma_{\mathrm{ess}}(A) \quad, \quad \sigma_{\mathrm{ess}}(A) \subset \sigma_{\mathrm{ess}}\left(A_{\infty}\right) .\right.\right.\right.\right.
$$

In fact, the difficulties due to the non ellipticity of $A$ appear only in the proof of the last inclusion, making it slightly more complicated than the two first ones. 


\section{Lemma 3.3.}

$$
\sigma\left(A_{\infty}\right) \subset\left[\gamma_{\mathrm{TM}},+\infty[.\right.
$$

Proof. From the definition of $\gamma_{\mathrm{TM}}$, we have for almost every $x_{1} \in \mathbb{R}$ and every $u \in D\left(A_{\infty}\right)$ :

$$
\int_{\mathbb{R}^{+}} \frac{1}{\varepsilon_{\infty}}\left(\left|\frac{\mathrm{d} u}{\mathrm{~d} x_{2}}\right|^{2}+\beta^{2}|u|^{2}\right) \mathrm{d} x_{2} \geq \gamma_{T M} \int_{\mathbb{R}^{+}}|u|^{2} \mathrm{~d} x_{2} .
$$

Integrating this inequality with respect to $x_{1}$, one obtains that:

$$
\left(A_{\infty} u, u\right)=\int_{\Omega} \frac{1}{\varepsilon_{\infty}}\left(\left|\frac{\mathrm{d} u}{\mathrm{~d} x_{2}}\right|^{2}+\beta^{2}|u|^{2}\right) \mathrm{d} x_{1} \mathrm{~d} x_{2} \geq \gamma_{\mathrm{TM}}\|u\|^{2}, \quad \forall u \in D\left(A_{\infty}\right) .
$$

Since $A_{\infty}$ is self-adjoint, we have thus:

$$
\inf \sigma\left(A_{\infty}\right)=\inf _{u \in D\left(A_{\infty}\right), u \neq 0} \frac{\left(A_{\infty} u, u\right)}{\|u\|^{2}} \geq \gamma_{\mathrm{TM}},
$$

and the lemma is proved.

Let us now prove the second inclusion of (12).

\section{Lemma 3.4.}

$$
\left[\gamma_{\mathrm{TM}},+\infty\left[\subset \sigma_{\mathrm{ess}}(A) .\right.\right.
$$

Proof. We are going to show that we can associate to every $\lambda \geq \gamma_{T M}$ a singular sequence of $A$. To achieve this, we introduce the function:

$$
k \longrightarrow \Gamma_{\mathrm{TM}}(k)=\inf _{u \in H^{1}\left(\mathbb{R}^{+}\right), u \neq 0} \frac{\int_{0}^{+\infty} \frac{1}{\varepsilon_{\infty}\left(x_{2}\right)}\left(\left|\frac{\mathrm{d} u}{\mathrm{~d} x_{2}}\right|^{2}+k^{2}|u|^{2}\right) \mathrm{d} x_{2}}{\int_{0}^{+\infty}|u|^{2} \mathrm{~d} x_{2}},
$$

such that: $\Gamma_{\mathrm{TM}}(\beta)=\gamma_{\mathrm{TM}}$. Then, we notice that the function:

$$
\xi \longrightarrow \Gamma_{\mathrm{TM}}\left(\sqrt{\beta^{2}+\xi^{2}}\right)
$$

is continuous and strictly increasing on $\mathbb{R}^{+}$, and is thus bijective from $\mathbb{R}^{+}$onto $\left[\Gamma_{\mathrm{TM}}(\beta),+\infty\left[=\left[\gamma_{\mathrm{TM}},+\infty[\right.\right.\right.$. Consequently, for every $\lambda \geq \gamma_{\mathrm{TM}}$, there exists a unique $\xi \geq 0$ such that:

$$
\lambda=\Gamma_{\mathrm{TM}}(k), \quad k=\sqrt{\beta^{2}+\xi^{2}} .
$$

To build up a singular sequence associated to $\lambda$, we use the planar wave-guide fundamental mode $u_{k}$ associated to $\Gamma_{\mathrm{TM}}(k)$. More precisely, $u_{k}$ satisfies:

$$
\left\{\begin{array}{l}
\int_{0}^{+\infty}\left|u_{k}\left(x_{2}\right)\right|^{2} \mathrm{~d} x_{2}=1 \\
-\frac{\mathrm{d}}{\mathrm{d} x_{2}}\left(\frac{1}{\varepsilon_{\infty}} \frac{\mathrm{d} u_{k}}{\mathrm{~d} x_{2}}\right)+\frac{k^{2}}{\varepsilon_{\infty}} u_{k}=\Gamma_{\mathrm{TM}}(k) u_{k} \\
\frac{\mathrm{d} u_{k}}{\mathrm{~d} x_{2}}(0)=0
\end{array}\right.
$$


Let $\theta \in \mathcal{C}^{\infty}(\mathbb{R})$ such that $\theta_{\mid[-\ell / 2, \ell / 2]}=0$ (recall that $\ell$ is the width of the strip) and $\int_{-\infty}^{+\infty}\left|\theta\left(x_{1}\right)\right|^{2} \mathrm{~d} x_{1}=1$. Consider the sequence $u_{n}$ of $H^{1}(\Omega)$ defined by:

$$
u_{n}(x)=\theta_{n}\left(x_{1}\right) \mathrm{e}^{i \xi x_{1}} u_{k}\left(x_{2}\right),
$$

where:

$$
\theta_{n}\left(x_{1}\right)=\frac{1}{\sqrt{n}} \theta\left(\frac{x_{1}}{n}\right)
$$

One can easily check that:

$$
\left\{\begin{array}{l}
\left\|u_{n}\right\|_{L^{2}}=1, \\
u_{n} \rightarrow 0 \quad \text { dans } L^{2}(\Omega), \\
u_{n} \in D(A) .
\end{array}\right.
$$

In addition, the second equation of (13) shows that we have in $\Omega_{D}$ and $\Omega_{A}$ :

$$
\begin{aligned}
A u_{n} & =\theta_{n} \mathrm{e}^{i \xi x_{1}} \frac{1}{\varepsilon_{\infty}}\left(-\frac{\mathrm{d}^{2} u_{k}}{\mathrm{~d} x_{2}^{2}}+k^{2} u_{k}\right)-\frac{2}{\varepsilon_{\infty}} i \xi \frac{\mathrm{d} \theta_{n}}{\mathrm{~d} x_{1}} \mathrm{e}^{i \xi x_{1}} u_{k}-\frac{1}{\varepsilon_{\infty}} \frac{\mathrm{d}^{2} \theta_{n}}{\mathrm{~d} x_{1}^{2}} \mathrm{e}^{i \xi x_{1}} u_{k} \\
& =\lambda u_{n}-\frac{2}{\varepsilon_{\infty}} i \xi \frac{\mathrm{d} \theta_{n}}{\mathrm{~d} x_{1}} \mathrm{e}^{i \xi x_{1}} u_{k}-\frac{1}{\varepsilon_{\infty}} \frac{\mathrm{d}^{2} \theta_{n}}{\mathrm{~d} x_{1}^{2}} \mathrm{e}^{i \xi x_{1}} u_{k} .
\end{aligned}
$$

Since $\frac{\mathrm{d} \theta_{n}}{\mathrm{~d} x_{1}}$ and $\frac{\mathrm{d}^{2} \theta_{n}}{\mathrm{~d} x_{1}^{2}}$ converge strongly to zero in $L^{2}(\mathbb{R})$, we have thus: $\left\|A u_{n}-\lambda u_{n}\right\|_{L^{2}} \longrightarrow 0$. Consequently, $\lambda=\Gamma_{\mathrm{TM}}\left(\sqrt{\beta^{2}+\xi^{2}}\right)$ belongs to $\sigma_{\mathrm{ess}}(A)$ for every $\xi \geq 0$, and thus $\left[\Gamma_{\mathrm{TM}}(\beta),+\infty\left[=\left[\gamma_{\mathrm{TM}},+\infty\left[\subset \sigma_{\mathrm{ess}}(A)\right.\right.\right.\right.$.

To conclude the proof of Theorem 3.1, let us prove the last and most difficult inclusion:

\section{Lemma 3.5.}

$$
\sigma_{\text {ess }}(A) \subset \sigma_{\text {ess }}\left(A_{\infty}\right) .
$$

Proof. Let $\lambda \in \sigma_{\text {ess }}(A)$ and $u_{n} \in D(A)$ be a singular sequence associated to $\lambda$ :

$$
\left\{\begin{array}{l}
\left\|u_{n}\right\|_{L^{2}}=1, \\
u_{n} \rightarrow 0 \quad \text { in } L^{2}(\Omega), \\
\left\|A u_{n}-\lambda u_{n}\right\|_{L^{2}} \longrightarrow 0 .
\end{array}\right.
$$

In order to build up a singular sequence of $A_{\infty}$ associated to $\lambda$, set: $v_{n}=\theta u_{n}$ where $\theta \in \mathcal{C}_{0}^{\infty}(\Omega)$ satifies:

- $\forall x \in \Omega_{\infty}: 0 \leq \theta(x) \leq 1$.

- There exist two open sets $\mathcal{O}$ and $\mathcal{O}^{\prime}$ of $\Omega$ such that:

$$
\left\{\begin{array}{l}
\Omega_{S} \subset \mathcal{O} \subset \mathcal{O}^{\prime}, \\
\theta=0 \quad \text { in } \mathcal{O}, \\
\theta=1 \quad \text { in } \Omega \backslash \mathcal{O}^{\prime} .
\end{array}\right.
$$

- $\theta\left(x_{1}, x_{2}\right)=\theta\left(x_{1}\right)$ in the vicinity of the interface $\Sigma=\left\{x_{2}=h\right\}$ between the dielectric and the air. 
We shall prove that $v_{n}$ is a singular sequence of $A_{\infty}$ associated to $\lambda$.

First, it is clear that $v_{n}$ belongs to the domain $D\left(A_{\infty}\right)$ of $A_{\infty}$, and that it converges weakly to zero in $L^{2}(\Omega)$. To conclude, it remains to prove that:

(i) $v_{n}$ does not converge (strongly) to zero in $L^{2}(\Omega)$.

(ii) $A_{\infty} v_{n}-\lambda v_{n}$ converges to zero in $L^{2}(\Omega)$.

These results will follow from the properties of operator $A$ stated below.

Theorem 2.4 shows that operator $A$ defines an isomorphism from $D(A)$ onto $L^{2}(\Omega)$. As a consequence, there exists $C>0$ such that:

$$
\|u\|_{H^{1}(\Omega)} \leq C\|A u\|_{L^{2}}, \quad \forall u \in D(A)
$$

Furthermore, classical regularity results for elliptic transmission problems show that $u$ has an $H^{2}$-regularity on both sides of the interface $\Sigma \backslash(\Sigma \cap \Gamma)$ between the air and the dielectric substrate. Consequently, if $\mathcal{U}$ is an open set such that $\mathcal{U} \cap \Gamma=\emptyset$, the following estimate holds:

$$
\|u\|_{H^{2}\left(\mathcal{U} \cap \Omega_{D}\right)}+\|u\|_{H^{2}\left(\mathcal{U} \cap \Omega_{A}\right)} \leq C\|A u\|_{L^{2}, \quad \forall u \in D(A) .}
$$

(i) Applying these results to the sequence $u_{n}$, one obtains that:

$$
\left\|u_{n}\right\|_{H^{1}(\Omega)} \leq C\left\|A u_{n}\right\|_{L^{2}}
$$

On the other hand, since: $\left(A u_{n}, u_{n}\right)=\left(A u_{n}-\lambda u_{n}, u_{n}\right)+\lambda\left\|u_{n}\right\|_{L^{2}}^{2}$, the sequence $A u_{n}$ is bounded in $L^{2}(\Omega)$. Inequality (15) shows that $u_{n}$ converges (up to a subsequence) to zero in $L_{\mathrm{loc}}^{2}(\Omega)$. Consequently, the function $1-\theta$ having a compact support, we have:

$$
\left\|v_{n}\right\|_{L^{2}}=\left\|\theta u_{n}\right\|_{L^{2}}=\left\|u_{n}-(1-\theta) u_{n}\right\|_{L^{2}} \longrightarrow 1
$$

and point (i) is thus proved.

(ii) A straightforward computation shows that:

$$
A_{\infty} v_{n}-\lambda v_{n}=\theta\left(A u_{n}-\lambda u_{n}\right)-\frac{1}{\varepsilon_{\infty}}\left(u_{n} \triangle \theta+2 \nabla \theta \cdot \nabla u_{n}\right)
$$

Since $\triangle \theta$ has a compact support and since the sequence $u_{n}$ converges to zero in $L_{\text {loc }}^{2}(\Omega)$, we have:

$$
\lim _{n \longrightarrow+\infty}\left\|u_{n} \triangle \theta\right\|_{L^{2}}=0 .
$$

If $\mathcal{U}$ denotes the support of $\nabla \theta$, the regularity results recalled above show that:

$$
\left\|u_{n}\right\|_{H^{2}\left(\mathcal{U} \cap \Omega_{D}\right)}+\left\|u_{n}\right\|_{H^{2}\left(\mathcal{U} \cap \Omega_{A}\right)} \leq C\left\|A u_{n}\right\|_{L^{2}} .
$$

Thus, the sequence $u_{n}$ converges to zero in $H^{1}(\mathcal{U})$ and consequently:

$$
\lim _{n \rightarrow+\infty}\left\|\nabla \theta \cdot \nabla u_{n}\right\|_{L^{2}}=0 .
$$

As a consequence:

$$
\lim _{n \longrightarrow+\infty}\left\|A_{\infty} v_{n}-\lambda v_{n}\right\|_{L^{2}}=0
$$

and $v_{n}$ is a singular sequence of $A_{\infty}$ associated to $\lambda$. 


\section{Negative Eigenvalues}

In this section, we are going to see that $A$ has a sequence of negative eigenvalues that converges to $-\infty$. This property is directly related to the negative value of the dielectric permittivity in the superconducting strip.

Let us start by proving the following preliminary result:

Proposition 4.1. Operator $A$ is not bounded from below.

Proof. To prove this proposition, we construct a sequence $u_{n} \in D(A)$ satisfying:

$$
\left\{\begin{array}{l}
\left\|u_{n}\right\|_{L^{2}}=1, \forall n \geq 0 \\
\lim _{n \longrightarrow+\infty}\left(A u_{n}, u_{n}\right)=-\infty .
\end{array}\right.
$$

Let $x_{0} \in \Omega_{S}$ and $\delta>0$ be such that the ball $\mathcal{B}=\left\{x,\left|x-x_{0}\right|<\delta\right\}$ is contained in $\Omega_{S}$. We define then the sequence:

$$
u_{n}(x)=n \theta\left(n\left|x-x_{0}\right|\right),
$$

where $\theta$ is a regular radial function with support in $\mathcal{B}$, such that $\|\theta\|_{L^{2}}=1$.

Since Supp $u_{n} \subset\left\{\left|x-x_{0}\right|<\delta / n\right\} \subset \Omega_{S}$, we have $u_{n} \in D(A)$. Furthermore:

$$
\left\|u_{n}\right\|_{L^{2}}=1 \quad \text { and } \quad\left\|\nabla u_{n}\right\|_{L^{2}}=n\|\nabla \theta\|_{L^{2}} .
$$

As a consequence:

$$
\left(A u_{n}, u_{n}\right)=\frac{1}{\varepsilon_{S}} \int_{\Omega_{S}}\left(\left|\nabla u_{n}\right|^{2}+\beta^{2}\left|u_{n}\right|^{2}\right)=\frac{1}{\varepsilon_{S}}\left(n^{2}\|\nabla \theta\|_{L^{2}}^{2}+\beta^{2}\right) \longrightarrow-\infty .
$$

We immediately deduce from this proposition the:

Corollary 4.2. Operator $A$ admits a sequence of negative eigenvalues tending to $-\infty$.

Proof. Since $A$ is self-adjoint, we have:

$$
\inf \sigma(A)=\inf _{u \in D(A), u \neq 0} \frac{(A u, u)}{\|u\|_{L^{2}}},
$$

where $\sigma(A)$ denotes the spectrum of $A$. Thus, it follows from Proposition 4.1 that: $\inf \sigma(A)=-\infty$. On the other hand, if $\sigma_{p}(A)$ denotes the point spectrum of $A$, which is composed of isolated eigenvalues of finite multiplicity, we have:

$$
\sigma(A)=\sigma_{\mathrm{ess}}(A) \cup \sigma_{p}(A)=\left[\gamma_{\mathrm{TM}},+\infty\left[\cup \sigma_{p}(A) .\right.\right.
$$

Consequently: $\inf \sigma_{p}(A)=-\infty$. This shows the existence of a sequence $\lambda_{n}$ of negative eigenvalues of $A$ satisfying: $\lim _{n \longrightarrow+\infty} \lambda_{n}=-\infty$.

We shall now make use of these results concerning the spectrum of $A$ to prove the existence of positive eigenvalues of $A$. 


\section{Positive eigenvalues}

Applying the Min-Max principle to operator $A^{-1}$, we derive in the first part of this section a necessary and sufficient condition for the existence of positive eigenvalues. In the second part, we use the condition previously derived to prove existence results for positive eigenvalues of $A$.

\subsection{Characterization of the positive eigenvalues of $A$}

The Min-Max principle ( $c f$. [14]) provides a criterion to prove existence of eigenvalues located below the essential spectrum for self-adjoint bounded from below operators. More precisely, let $B$ be such an operator with domain $D(B) \subset L^{2}(\Omega), b(\cdot, \cdot)$ the bilinear form with domain $D(b)$ associated to it, and $\gamma_{\text {ess }}=\inf \sigma_{\text {ess }}(B)$.

For $m \geq 1$, we introduce the real numbers $\lambda_{m}$ equivalently defined by the two formulas:

$$
\lambda_{m}=\inf _{V_{m} \in \mathcal{V}_{m}(D(B))} \sup _{u \in V_{m}, u \neq 0} \frac{(B u, u)}{\|u\|_{L^{2}}^{2}}=\inf _{V_{m} \in \mathcal{V}_{m}(D(b))} \sup _{u \in V_{m}, u \neq 0} \frac{b(u, u)}{\|u\|_{L^{2}}^{2}}
$$

where $\mathcal{V}_{m}(V)$ denotes the set of all $m$-dimensional subspaces of $V$.

The Min-Max principle states that operator $B$ has at least $m$ eigenvalues $\lambda$ satisfying $\lambda<\gamma_{\text {ess }}$ if and only if $\lambda_{m}<\gamma_{\text {ess }}$. In other words, if $\mathcal{N}$ denotes the number of such eigenvalues, the following assertion holds:

$$
\lambda_{m}<\gamma_{\text {ess }} \Longleftrightarrow \mathcal{N} \geq m
$$

Furthermore, if $\mathcal{N} \geq m$, the $m$ first eigenvalues of $B$ are exactly the numbers $\lambda_{p}, 1 \leq p \leq m$.

In the case studied here, operator $A$ is neither bounded from below (because of its infinite sequence of negative eigenvalues, cf. Prop. 4.1), and of course, nor from above. Consequently, the Min-Max principle cannot be applied to it. We have thus to find a new criterion for existence of eigenvalues. Moreover, we have an extra constraint concerning the construction of this criterion: it must be sharp enough to prove the existence of positive eigenvalues for $A$, since these eigenvalues are the only ones of interest from the physical point of view. To achieve this, the solution we propose is to apply the Min-Max principle to the operator $A^{-1}$ (recall that condition (8) is supposed to be satisfied and that $A$ is thus invertible).

Since $\lambda \in \sigma(A)$ if and only if $\lambda^{-1} \in \sigma\left(A^{-1}\right)$, Theorem 3.1 and Corollary 4.2 lead to:

Proposition 5.1. Operator $A^{-1}$ is self-adjoint, bounded from below and from above. Moreover, $A^{-1}$ has a sequence of negative eigenvalues tending to zero, and its continuous spectrum is $\left.] 0,\left(\gamma_{\mathrm{TM}}\right)^{-1}\right]$.

Figures 2 and 3 below show the structure of the spectra of $A$ and $A^{-1}$. As it can be seen from these figures, we have:

$$
\lambda \in \sigma_{p}(A) \cap \mathbb{R}^{+} \quad \Leftrightarrow \quad \mu=1 / \lambda \in \sigma_{p}\left(A^{-1}\right) \quad \text { and } \quad \mu>1 / \gamma_{\mathrm{TM}} .
$$

Consequently, the determination of the positive eigenvalues of $A$ amounts to the determination of the eigenvalues of $A^{-1}$ located above its essential spectrum. Applying the Min-Max principle to the self-adjoint and bounded from below operator $B=-A^{-1}$, we obtain the following result:

Theorem 5.2. Let $\mathcal{N}$ be the number of positive eigenvalues $\lambda$ of $A$ satisfying $\lambda<\gamma_{\mathrm{TM}}$ (counted with their multiplicity), and define:

$$
\mu_{m}=\sup _{V_{m} \in \mathcal{V}_{m}(D(A))} \inf _{u \in V_{m}, u \neq 0} \frac{(A u, u)}{\|A u\|_{L^{2}}^{2}} .
$$

Then, for all $m \geq 1$ :

$$
\mathcal{N} \geq m \quad \Leftrightarrow \quad \mu_{m}>\frac{1}{\gamma_{\mathrm{TM}}}
$$




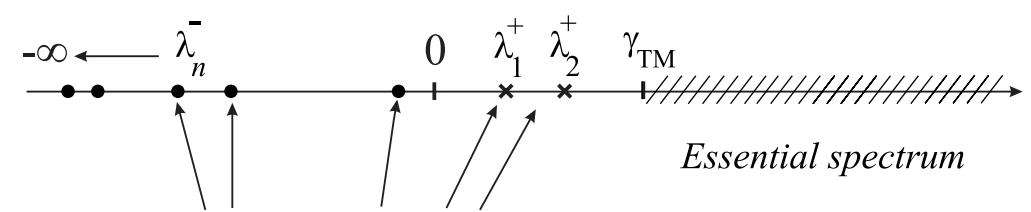

Point spectrum

Figure 2. Spectrum of $A$.

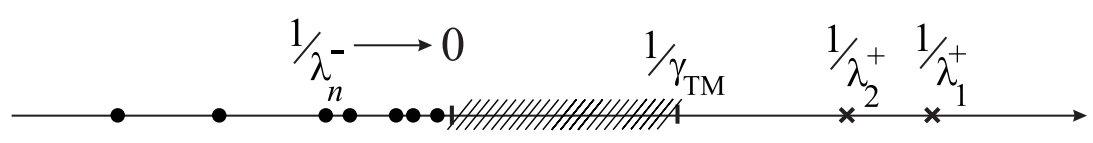

Essential spectrum

FiguRE 3. Spectrum of $A^{-1}$.

In addition, if $\mathcal{N} \geq m$, the first $m$ eigenvalues $\lambda_{p}, p=1, . ., m$, of $A$ are given by the relation:

$$
\lambda_{p}=\frac{1}{\mu_{p}} .
$$

Proof. Denote by $\lambda_{m}(B)$ the min-max of order $m$ of $B=-A^{-1}$, and by $\mathcal{N}(B)$ the number of its eigenvalues which are smaller than the lower bound $-\frac{1}{\gamma_{\mathrm{TM}}}$ of its essentiel spectrum. Applying the Min-Max principle to the self-adjoint bounded from below operator $B$, we obtain that:

$$
\mathcal{N}(B) \geq m \quad \Leftrightarrow \quad \lambda_{m}(B)<-\frac{1}{\gamma_{\mathrm{TM}}},
$$

and that, if $\mathcal{N}(B) \geq m$, the numbers $\lambda_{p}(B), p=1, \ldots, m$ are exactly the $m$ first eigenvalues of $B$.

On the other hand, using the min-max formula, we have:

$$
\begin{aligned}
\lambda_{m}(B) & =\inf _{V_{m} \in \mathcal{V}_{m}\left(L^{2}(\Omega)\right)} \sup _{f \in V_{m}, f \neq 0} \frac{\left(-A^{-1} f, f\right)}{\|f\|_{L^{2}}^{2}} \\
& =-\sup _{V_{m} \in \mathcal{V}_{m}\left(L^{2}(\Omega)\right)} \inf _{f \in V_{m}, f \neq 0} \frac{\left(A^{-1} f, f\right)}{\|f\|_{L^{2}}^{2}} \\
& =-\sup _{V_{m} \in \mathcal{V}_{m}(D(A))} \inf _{u \in V_{m}, u \neq 0} \frac{(A u, u)}{\|A u\|_{L^{2}}^{2}} \\
& =-\mu_{m} .
\end{aligned}
$$

Furthermore, $\mathcal{N}(B)$ is nothing but $\mathcal{N}$. The equivalence (21) shows thus that:

$$
\mathcal{N} \geq m \quad \Leftrightarrow \quad \mu_{m}>\frac{1}{\gamma_{T M}}
$$

Finally, since the numbers $\lambda_{p}(B)=-\mu_{p}$, for $p=1, \ldots, m$ are the $m$ first eigenvalues of $B=-A^{-1}$, the positive real numbers $\left(\mu_{p}^{-1}\right)_{p=1, \ldots, m}$ are the $m$ first positive ones of $A$. 


\section{Remark 5.3.}

(i) The existence criterion (20) for positive eigenvalues can also be written:

$$
\mathcal{N} \geq m \quad \Leftrightarrow \quad\left\{\begin{array}{c}
\exists V_{m} \in \mathcal{V}_{m}(D(A)), \text { such that } \forall u \in V_{m} \\
\gamma_{\mathrm{TM}}(A u, u)>\|A u\|_{L^{2}}^{2}
\end{array}\right.
$$

(ii) One may notice that the criterion obtained imposes that the test functions of $V_{m}$ belong to the domain $D(A)$ of the operator, and satisfy in particular the suitable transmission conditions at the interfaces. This constraint makes the construction of such test functions much more complicated than in the usual case of an operator bounded from below. Indeed, in this last case, the $m$-dimensional subspaces $V_{m}$ are not included in the domain of the operator, but in the one of the bilinear form associated to it.

We shall now use the characterization obtained to prove existence results for positive eigenvalues $\lambda$ of $A$ satisfying $\lambda<\gamma_{\mathrm{TM}}$.

\subsection{Existence results for positive eigenvalues}

In this section, we are going to show how the criterion derived in Section 5.1 can be used to prove the existence of positive eigenvalues of the operator $A$. As stated in the point $(i)$ of Remark 5.3, operator $A$ admits at least $m$ positives eigenvalues smaller than $\gamma_{\mathrm{TM}}$ if and only if there exists an $m$-dimensional subspace $V_{m}$ of $D(A)$ such that:

$$
\gamma_{\mathrm{TM}}(A u, u)>\|A u\|_{L^{2}}^{2}, \quad \forall u \in V_{m} .
$$

We will see that the existence of such a subspace can be proved if the strip is wide enough, and its permittivity large enough (in modulus). This result is based on the fact that for large strips, the problem is "close" to the one-dimensional problem corresponding to the case of an infinitely wide strip. On the other hand, and as we shall see in the next section, this problem can be studied almost explicitely (see [6]), and has at least one positive eigenvalue for $\left|\varepsilon_{S}\right|$ large enough.

\subsubsection{The one-dimensional model (strip of infinite width)}

This model is associated to the unbounded operator $A^{1}$ of $L^{2}\left(\mathbb{R}^{+}\right)$, defined by:

$$
\left\{\begin{array}{l}
D\left(A^{1}\right)=\left\{u \in H^{1}\left(\mathbb{R}^{+}\right), \frac{\mathrm{d}}{\mathrm{d} x_{2}}\left(\frac{1}{\varepsilon} \frac{\mathrm{d} u}{\mathrm{~d} x_{2}}\right) \in L^{2}\left(\mathbb{R}^{+}\right), \frac{\mathrm{d} u}{\mathrm{~d} x_{2}}(0)=0\right\} \\
A^{1} u=-\frac{\mathrm{d}}{\mathrm{d} x_{2}}\left(\frac{1}{\varepsilon} \frac{\mathrm{d} u}{\mathrm{~d} x_{2}}\right)+\frac{\beta^{2}}{\varepsilon} u
\end{array}\right.
$$

where, for the sake of simplicity, we still denote by $\varepsilon$ the function of $x_{2}$ defined by:

$$
\varepsilon\left(x_{2}\right)= \begin{cases}\varepsilon_{D}>1 & 0<x_{2}<h \\ \varepsilon_{S}<0 & h<x_{2}<h+d \\ \varepsilon_{A}=1 & x_{2}>h+d .\end{cases}
$$

One can then easily check (see [6]) that $A^{1}$ is self-adjoint for all the values of $\varepsilon_{S}$, has a sequence of negative eigenvalues tending to $-\infty$, and that its essential spectrum is $\left[\beta^{2},+\infty[\right.$. Furthermore, we have the following existence result for the first positive eigenvalue:

Proposition 5.4. There exists $\varepsilon_{S}^{1}<0$ such that for $\varepsilon_{S}<\varepsilon_{S}^{1}$, $A^{1}$ has (at least) one positive eigenvalue $\lambda_{1}<\beta^{2}$. Furthermore, this eigenvalue satisfies: $\lambda_{1}<\gamma_{\mathrm{TM}}$. 
Proof. The characterization of positive eigenvalues given in Theorem 5.2 can be applied to the operator $A^{1}$ studied here. Consequenlty, $\lambda_{1}$ exists if and only if there exists a test fuction $u \in D\left(A^{1}\right)$ such that:

$$
\beta^{2}\left(A^{1} u, u\right)>\left\|A^{1} u\right\|_{L^{2}}^{2} .
$$

Let then $u^{1}$ be a given function of $x_{2}$ satisfying (recall that $h$ and $d$ denote respectively the thickness of the dielectric and the thickness of the strip):

$$
\left\{\begin{array}{c}
u^{1} \in \mathcal{C}^{\infty}([0,+\infty[), \\
u_{\mid[0, h]}^{1}=1 \quad \text { and } \quad u_{\mid[h+d,+\infty[}^{1}=0 .
\end{array}\right.
$$

Since $u^{1}$ and $\frac{1}{\varepsilon}\left(u^{1}\right)^{\prime}=\frac{1}{\varepsilon} \frac{\mathrm{d} u^{1}}{\mathrm{~d} x_{2}}$ are clearly continuous in $x_{2}=h$ and $x_{2}=h+d$, we have $u^{1} \in D\left(A^{1}\right)$. Furthermore, one can easily check that:

$$
\frac{\left(A^{1} u^{1}, u^{1}\right)}{\left\|A^{1} u^{1}\right\|_{L^{2}}^{2}}=\frac{\left(\beta^{2} / \varepsilon_{D}\right) h+a / \varepsilon_{S}}{\left(\beta^{2} / \varepsilon_{D}\right)^{2} h+b / \varepsilon_{S}^{2}}
$$

where we have set:

$$
a=\int_{h}^{h+d}\left|\left(u^{1}\right)^{\prime}\right|^{2}+\beta^{2}\left|u^{1}\right|^{2} \quad \text { and } \quad b=\int_{h}^{h+d}\left|-\left(u^{1}\right)^{\prime \prime}+\beta^{2} u^{1}\right|^{2} .
$$

Thus:

$$
\lim _{\varepsilon_{S} \longrightarrow-\infty} \frac{\left(\beta^{2} / \varepsilon_{D}\right) h+a / \varepsilon_{S}}{\left(\beta^{2} / \varepsilon_{D}\right)^{2} h+b / \varepsilon_{S}^{2}}=\varepsilon_{D} / \beta^{2} .
$$

But as it has been said in Remark 3.2, we have:

$$
\varepsilon_{D} / \beta^{2}>1 / \gamma_{\mathrm{TM}}>1 / \beta^{2} .
$$

Thus, there exists $\varepsilon_{S}^{1}<0$ such that:

$$
\frac{\left(A^{1} u^{1}, u^{1}\right)}{\left\|A^{1} u^{1}\right\|_{L^{2}\left(\mathbb{R}^{+}\right)}^{2}}>\frac{1}{\gamma_{\mathrm{TM}}}>\frac{1}{\beta^{2}}, \quad \forall \varepsilon_{S}<\varepsilon_{S}^{1} .
$$

This proves the existence of a positive eigenvalue $\lambda_{1}$ of $A^{1}$ satisfying: $\lambda_{1}<\gamma_{\mathrm{TM}}<\beta^{2}$.

\section{Remark 5.5.}

(i) Obviously, some sharper estimates of $\lambda_{1}$ can be obtained using more accurate test functions than $u^{1}$. This can be achieved thanks to the fact that the computations, although being heavy, are quiet explicit. In fact, our aim here was only to give an idea of the kind of methods one can use to prove existence of positive eigenvalues for the one-dimensional problem.

(ii) The fact that $\lambda_{1}$ satisfies $\lambda_{1}<\gamma_{\mathrm{TM}}$ will be useful in the next section, to prove existence results for the eigenvalues of the two-dimensional problem associated to $A$. 


\subsubsection{Back to the two-dimensional model}

The main result of this section is given by the following theorem (recall that $\ell$ is the width of the strip):

Theorem 5.6. For every $m \geq 1$, there exist $\varepsilon_{S}^{1}<0$ and $\ell_{m}>0$ such that the number $\mathcal{N}$ of positive eigenvalues $\lambda$ of $A$ such that $\lambda<\gamma_{\mathrm{TM}}$ satisfies:

$$
\forall \varepsilon_{S}<\varepsilon_{S}^{1} \quad, \quad \forall \ell \geq \ell_{m} \quad: \quad \mathcal{N} \geq m
$$

Proof. Theorem 5.2 shows that the result will be proved if there exist $\varepsilon_{S}^{1}<0$ and $\ell_{m}>0$ such that for every $\varepsilon_{S}<\varepsilon_{S}^{1}$ and every $\ell \geq \ell_{m}$, we can find an $m$-dimensional subspace $V_{m}$ of $D(A)$ such that:

$$
\gamma_{\mathrm{TM}}(A u, u)>\|A u\|_{L^{2}}^{2}, \quad \forall u \in V_{m}
$$

To construct such a subspace, we shall use the test function $u^{1}$ (satisfying (24)) we have used in the study of the one-dimensional problem (corresponding to $\ell=+\infty$ ).

Let us set:

$$
u_{p, n}=\frac{1}{\sqrt{n}} \theta_{p}\left(\frac{x_{1}}{n}\right) u^{1}\left(x_{2}\right) \quad \text { and } \quad V_{m}^{n}=\operatorname{Vect}\left\{u_{p, n}, p=1, . ., m\right\}
$$

where: $\theta_{1}, \theta_{2}, . ., \theta_{m} \in \mathcal{C}_{0}^{\infty}(]-1,1[)$ are supposed to be linearly independant.

Since $u^{1}$ satisfies the transmission conditions $\left[u^{1}\right]=\left[\frac{1}{\varepsilon} \frac{\mathrm{d} u^{1}}{\mathrm{~d} x_{2}}\right]=0$ at the interfaces and since $\operatorname{Supp}\left(u_{p, n}\right) \subset$ $[-n, n]$, we have:

$$
u_{p, n} \in D(A), \quad \forall \ell \geq n
$$

Thus:

$$
V_{m}^{n} \subset \mathcal{V}_{m}(D(A)), \quad \forall \ell \geq n
$$

Let then $u_{n}=\sum_{p=1}^{m} \mu_{p} u_{p, n} \in V_{m}^{n}$, with $\left(\mu_{1}, . ., \mu_{m}\right) \neq 0$. A straightforward computation shows that for $\ell \geq n$, we have:

where we have set:

$$
\frac{\left(A u_{n}, u_{n}\right)}{\left\|A u_{n}\right\|^{2}}=\frac{\left(A^{1} u^{1}, u^{1}\right)+\frac{\alpha^{\prime}}{\alpha} \frac{1}{n^{2}}\left(\int_{0}^{+\infty} \frac{1}{\varepsilon}\left|u^{1}\right|^{2}\right)}{\int_{0}^{+\infty}\left|A^{1} u^{1}\right|^{2}-2 \frac{\alpha^{\prime}}{\alpha} \frac{1}{n^{2}}\left(\int_{0}^{+\infty} \frac{1}{\varepsilon} u^{1} A^{1} u^{1}\right)+\frac{\alpha^{\prime \prime}}{\alpha} \frac{1}{n^{4}}\left(\int_{0}^{+\infty} \frac{1}{|\varepsilon|^{2}}\left|u^{1}\right|^{2}\right)}
$$

$$
\alpha=\int_{-\infty}^{+\infty}|\theta|^{2}, \quad \alpha^{\prime}=\int_{-\infty}^{+\infty}\left|\theta^{\prime}\right|^{2}, \quad \alpha^{\prime \prime}=\int_{-\infty}^{+\infty}\left|\theta^{\prime \prime}\right|^{2}, \quad \theta=\sum_{p=1}^{m} \mu_{p} \theta_{p}
$$

We shall prove that for $n$ and $\left|\varepsilon_{S}\right|$ large enough, we have:

$$
\frac{\left(A u_{n}, u_{n}\right)}{\left\|A u_{n}\right\|_{L^{2}}^{2}}>\frac{1}{\gamma_{\mathrm{TM}}}
$$

First, notice that the functionals $\alpha^{\prime} / \alpha$ and $\alpha^{\prime \prime} / \alpha$ are bounded on the linear subspace $V_{\theta}$ spanned by the functions $\left(\theta_{p}\right)_{p=1, . ., m}$, since $\alpha^{\prime}$ and $\alpha^{\prime \prime}$ are continuous on the compact subset of $V_{\theta}$ defined by $\int_{-\infty}^{+\infty}|\theta|^{2}=1$. Consequently, 
we have:

$$
\xi_{n}^{1} \leq \frac{\left(A u_{n}, u_{n}\right)}{\left\|A u_{n}\right\|_{L^{2}}^{2}} \leq \xi_{n}^{2}
$$

where $\xi_{n}^{1}$ et $\xi_{n}^{2}$ are two sequences (which do not depend on $\ell$ ) satisfying:

$$
\lim _{n \longrightarrow+\infty} \xi_{n}^{1}=\lim _{n \longrightarrow+\infty} \xi_{n}^{2}=\frac{\left(A^{1} u^{1}, u^{1}\right)}{\left\|A^{1} u^{1}\right\|_{L^{2}}^{2}} .
$$

It follows then from (25) and (27) that there exist $N>0$ and $\varepsilon_{S}^{1}<0$ such that for $\ell \geq N$, we have:

$$
u_{N} \in D(A) \quad \text { and } \quad \frac{\left(A u_{N}, u_{N}\right)}{\left\|A u_{N}\right\|_{L^{2}}^{2}}>\frac{1}{\gamma_{\mathrm{TM}}}, \quad \forall \varepsilon_{S}<\varepsilon_{S}^{1} .
$$

Thus inequality (26) holds, with $V_{m}=V_{m}^{N}$.

\section{The ASYMPtotic Limit $\varepsilon_{S} \longrightarrow-\infty$}

Making use of the Min-Max principle, we have shown in the previous section that for $\left|\varepsilon_{S}\right|$ large enough and for large superconducting strips, operator $A$ admits positive eigenvalues. In this section, we investigate the asymptotic behavior of the discrete spectrum of $A$ as $\varepsilon_{S}$ goes to $-\infty$. We will see that as expected from the physical context of the problem ( $c f$. Rem. 2.5), the limit case $\varepsilon_{S}=-\infty$ corresponds to the case of a perfectly conducting strip. If $A_{E}$ denotes the operator associated to this problem, we will show here that we can find in the neighborhood of every positive eigenvalue of $A_{E}$ at least one eigenvalue of $A$ for $\left|\varepsilon_{S}\right|$ large enough. The main advantage of this asymptotic result lies in the fact that contrary to $A$, operator $A_{E}$ is elliptic and thus its spectral analysis is much more standard than the one of $A$.

This section is organised as follows. In Section 6.1, the case of a perfectly conducting strip is studied. In Section 6.2 , we will see precisely in what sense the operator $A$ is close to $A_{E}$ for large values of $\left|\varepsilon_{S}\right|$. Finally, in Section 6.3 , we shall use the results obtained in the previous section to prove existence of positive eigenvalues for $A$, once again for $\left|\varepsilon_{S}\right|$ large enough.

\subsection{The perfectly conducting micro-strip line}

Here, the Neumann boundary condition is imposed on both the strip's and the ground's plane boundaries. The associated operator $A_{E}$ is thus defined as follows:

$$
\left\{\begin{array}{l}
D\left(A_{E}\right)=\left\{u \in H^{1}\left(\Omega_{E}\right), \operatorname{div}\left(\frac{1}{\varepsilon_{E}} \nabla u\right) \in L^{2}\left(\Omega_{E}\right), \partial_{n} u=0 \text { on } \Gamma \cup \Gamma_{P}\right\} \\
A_{E} u=-\operatorname{div}\left(\frac{1}{\varepsilon_{E}} \nabla u\right)+\frac{\beta^{2}}{\varepsilon_{E}} u
\end{array}\right.
$$

Since this operator is symmetric and elliptic, it is positive and self-adjoint. Furthermore, following the proof of Theorem 3.1, one can easily check that its continuous spectrum is the interval $] \gamma_{\mathrm{TM}},+\infty[$. Finally, using the Min-Max principle, some sufficient conditions (on the characteristics of the line) ensuring the existence of $m$ positive eigenvalues in $] 0, \gamma_{\mathrm{TM}}[$ can be derived very easily. Indeed, using for instance the $m$-dimensional 
subspace spanned by the $m$ functions $\left(u_{p}\right)_{p=1, . ., m}$ defined by:

$$
u_{p}\left(x_{1}, x_{2}\right)=\left\{\begin{array}{lc}
\sin \left(\frac{p \pi}{2 \ell}\left(2 x_{1}+\ell\right)\right) & \text { for }\left|x_{1}\right|<\ell / 2 \text { and } 0<x_{2}<d \\
0 & \text { elsewhere }
\end{array}\right.
$$

we obtain that the min-max of order $m$ satisfies:

$$
\lambda_{m} \leq \sup _{u \in V_{m}, u \neq 0} \frac{\left(A_{E} u, u\right)}{\|u\|_{L^{2}\left(\Omega_{E}\right)}^{2}}=\frac{\left(A_{E} u_{m}, u_{m}\right)}{\left\|u_{m}\right\|_{L^{2}\left(\Omega_{E}\right)}^{2}}=\frac{1}{\varepsilon_{D}}\left(\frac{m^{2} \pi^{2}}{\ell^{2}}+\beta^{2}\right) .
$$

Consequently, the Min-Max principle shows that $A_{E}$ has at least $m$ eigenvalues in $] 0, \gamma_{\mathrm{TM}}[$ as soon as the following condition is satisfied:

$$
\frac{1}{\varepsilon_{D}}\left(\frac{m^{2} \pi^{2}}{\ell^{2}}+\beta^{2}\right)<\gamma_{\mathrm{TM}}
$$

Note that for fixed values of $\varepsilon_{D}$ and $\beta$, this condition is satistfied for strips that are large enough.

To conclude this section, let us give a characterization of these positive eigenvalues of $A_{E}$ using the exterior Dirichlet to Neumann operator. This characterization will be very useful to compare the discrete spectra of $A$ and $A_{E}$ when $\varepsilon_{S} \longrightarrow-\infty$.

For $\lambda \in] 0, \gamma_{\mathrm{TM}}\left[\right.$ consider the operator $T_{E}(\lambda): H^{1 / 2}(\Gamma) \longrightarrow H^{-1 / 2}(\Gamma)$ defined for any trace $\varphi \in H^{1 / 2}(\Gamma)$ by:

$$
T_{E}(\lambda) \varphi=\partial_{n} v_{E \mid \Gamma},
$$

where $v_{E} \in H^{1}\left(\Omega_{E}\right)$ is the solution of the boundary value problem:

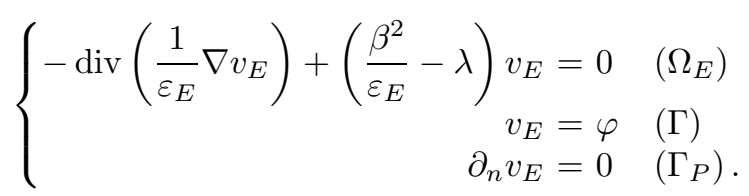

Before going further, let us emphasize that $T_{E}(\lambda)$ is well defined for every $\lambda \in\left[0, \gamma_{\mathrm{TM}}[\right.$. To check this, it suffices to prove that problem (30) is well-posed for such values of $\lambda$. Introducing the solution $w_{E} \in H^{1}\left(\Omega_{E}\right)$ of the well-posed boundary value problem:

$$
\left\{\begin{array}{rlrl}
-\operatorname{div}\left(\frac{1}{\varepsilon_{E}} \nabla w_{E}\right)+\frac{\beta^{2}}{\varepsilon_{E}} w_{E}=0 & & \left(\Omega_{E}\right) \\
w_{E} & =\varphi & & (\Gamma) \\
\partial_{n} w_{E} & =0 & & \left(\Gamma_{P}\right)
\end{array}\right.
$$

one sees that the well-posedness of (30) is equivalent to the well-posedness of the boundary value problem:

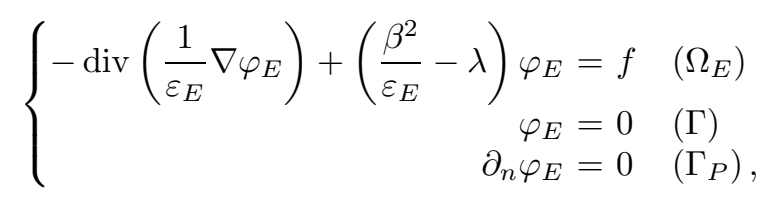

where we have set: $f=-\lambda w_{E} \in L^{2}\left(\Omega_{E}\right)$. 
Now, problem (31) has a unique solution if and only if the operator $\left(A_{E}^{D}-\lambda \mathrm{Id}\right)$ is invertible, where $A_{E}^{D}$ is the operator defined by:

$$
\left\{\begin{array}{l}
D\left(A_{E}^{D}\right)=\left\{u \in H^{1}\left(\Omega_{E}\right), \operatorname{div}\left(\frac{1}{\varepsilon_{E}} \nabla u\right) \in L^{2}\left(\Omega_{E}\right), u_{\mid \Gamma}=0, \partial_{n} u_{\mid \Gamma_{P}}=0\right\} \\
A_{E}^{D} u=-\operatorname{div}\left(\frac{1}{\varepsilon_{E}} \nabla u\right)+\frac{\beta^{2}}{\varepsilon_{E}} u .
\end{array}\right.
$$

The invertibility of $A_{E}^{D}$ for $\lambda \in\left[0, \gamma_{\mathrm{TM}}[\right.$ follows now immediately from:

\section{Lemma 6.1.}

$$
\sigma\left(A_{E}^{D}\right)=\sigma_{\mathrm{ess}}\left(A_{E}^{D}\right)=\left[\gamma_{\mathrm{TM}},+\infty[\right.
$$

Proof.

(i) For every $u \in D\left(A_{E}^{D}\right)$, we have:

$$
\left(A_{E}^{D} u, u\right)=\int_{\Omega_{E}} \frac{1}{\varepsilon_{E}}\left(|\nabla u|^{2}+\beta^{2}|u|^{2}\right)=\int_{\Omega} \frac{1}{\varepsilon_{\infty}}\left(|\nabla \tilde{u}|^{2}+\beta^{2}|\tilde{u}|^{2}\right)=\left(A_{\infty} \tilde{u}, \tilde{u}\right),
$$

where $\tilde{u}$ is the extension of $u$ to $\Omega$ by 0 (notice that $\tilde{u} \in H^{1}(\Omega)$ since $u \in H_{0}^{1}\left(\Omega_{E}\right)$ ) and $A_{\infty}$, defined by (9), is the operator corresponding to the unperturbed medium. It follows then from the study of $A_{\infty}$ (see Th. 3.1) that:

$$
\left(A_{E}^{D} u, u\right)=\left(A_{\infty} \tilde{u}, \tilde{u}\right) \geq \gamma_{\mathrm{TM}}\|\tilde{u}\|_{L^{2}(\Omega)}^{2}=\gamma_{\mathrm{TM}}\|u\|_{L^{2}\left(\Omega_{E}\right)}^{2},
$$

and thus (since $A_{E}^{D}$ is self-adjoint):

$$
\inf \sigma\left(A_{E}^{D}\right)=\inf _{u \in D\left(A_{E}^{D}\right), u \neq 0} \frac{\left(A_{E}^{D} u, u\right)}{\|u\|_{L^{2}\left(\Omega_{E}\right)}^{2}} \geq \gamma_{\mathrm{TM}}
$$

showing that:

$$
\sigma\left(A_{E}^{D}\right) \subset\left[\gamma_{\mathrm{TM}},+\infty[.\right.
$$

(ii) The converse inclusion can be proved by constructing for every $\lambda \geq \gamma_{\mathrm{TM}}$ a singular sequence of $A_{E}^{D}$ associated to $\lambda$.

Summing up, we have shown that for $\lambda \in\left[0, \gamma_{\mathrm{TM}}\left[\right.\right.$, problem (30) has a unique solution $v_{E} \in H^{1}\left(\Omega_{E}\right)$ for every $\varphi \in H^{1 / 2}(\Gamma)$, and thus that the operator $T_{E}(\lambda)$ such that $T_{E}(\lambda) \varphi=\partial_{n} v_{E \mid \Gamma}$ is well defined for such values of $\lambda$.

Remark 6.2. In fact, we have proved that $T_{E}(\lambda)$ is well defined on $\mathbb{C} \backslash\left[\gamma_{\mathrm{TM}},+\infty[\right.$.

Using this operator, one can see that $\lambda=\lambda_{E}$ is an eigenvalue of $A_{E}$ if and only if:

$$
\exists \varphi \in H^{1 / 2}(\Gamma) \quad \text { such that: } T_{E}\left(\lambda_{E}\right) \varphi=0 .
$$

In other words, we have the following characterization for the eigenvalues of $A_{E}$ in terms of $T_{E}(\lambda)$ :

Proposition 6.3. $\left.\lambda_{E} \in\right] 0, \gamma_{\mathrm{TM}}\left[\right.$ is eigenvalue of $A_{E}$ if and only if $\xi=\lambda_{E}$ is a pole of the operator-valued fonction $T_{E}(\xi)^{-1}$ of the complex variable $\xi$. 


\subsection{Asymptotic spectral analysis of $A$ : a boundary formulation}

Let us first introduce some notations needed in the sequel. We define the positive real parameter:

$$
\delta=-1 / \varepsilon_{S} .
$$

Furthermore, operator $A$ will be noted $A_{\delta}$ throughout this section. Our aim here is to prove that for small values of $\delta$, the eigenvalue problems associated to $A_{\delta}$ and $A_{E}$ are "close". The main difficulty in defining rigourously this property comes from the fact that the operators $A_{\delta}$ and $A_{E}$ do not have the same domains. To overcome this difficulty, we use the boundary formulation (on $\Gamma$ ) of the two eigenvalue problems, obtained thanks to the Dirichlet to Neumann operators.

Let us start by writing the eigenvalue problem $A_{\delta} u=\lambda u$ as an equivalent problem satisfied by the new unknown: $\varphi=u_{\mid \Gamma}$. First, note that:

$$
A_{\delta} u=\lambda u \Leftrightarrow\left\{\begin{aligned}
-\delta \triangle u_{S}+\left(\delta \beta^{2}+\lambda\right) u_{S} & =0 \\
-\operatorname{div}\left(\frac{1}{\varepsilon_{E}} \nabla u_{E}\right)+\left(\frac{\beta^{2}}{\varepsilon_{E}}-\lambda\right) u_{E} & =0 \\
u_{S}-u_{E} & =0 \\
\delta \partial_{n} u_{S}+\frac{1}{\varepsilon_{E}} \partial_{n} u_{E} & =0 \\
\partial_{n} u_{E} & =0
\end{aligned}\right.
$$

As a consequence, $\lambda \in] 0, \gamma_{\mathrm{TM}}\left[\right.$ is an eigenvalue of $A_{\delta}$ if and only if there exists $\varphi=u_{S \mid \Gamma}=u_{E \mid \Gamma} \in H^{1 / 2}(\Gamma)$ such that:

$$
T(\delta, \lambda) \varphi:=\left(\delta T_{S}(\delta, \lambda)+T_{E}(\lambda)\right) \varphi=0
$$

where $T_{E}(\lambda)$ is defined by $(29)$ and $(30)$, and $T_{S}(\delta, \lambda): H^{1 / 2}(\Gamma) \longrightarrow H^{-1 / 2}(\Gamma)$ is defined by:

$$
T_{S}(\delta, \lambda) \varphi=\partial_{n} v_{S \mid \Gamma}^{\delta}
$$

where $v_{S}^{\delta} \in H^{1}\left(\Omega_{S}\right)$ is the unique solution of the Dirichlet boundary value problem:

$$
\left\{\begin{aligned}
-\delta \triangle v_{S}^{\delta}+\left(\delta \beta^{2}+\lambda\right) v_{S}^{\delta}=0 & \left(\Omega_{S}\right) \\
v_{S}^{\delta}=\varphi & (\Gamma) .
\end{aligned}\right.
$$

Using the boundary operators $T_{S}(\delta, \lambda)$ and $T_{E}(\lambda)$, we have the following characterization of the eigenvalues of $A_{\delta}$ :

Proposition 6.4. The real number $\lambda \in] 0, \gamma_{\mathrm{TM}}\left[\right.$ is an eigenvalue of $A_{\delta}$ if and only if $\xi=\lambda$ is a pole of the operator-valued fonction:

$$
\xi \longrightarrow T(\delta, \xi)^{-1}=\left(\delta T_{S}(\delta, \xi)+T_{E}(\xi)\right)^{-1}
$$

The next step is to prove that operator $T(\delta, \lambda)=\delta T_{S}(\delta, \lambda)+T_{E}(\lambda)$ converges to $T_{E}(\lambda)$ (in the space of continuous operators from $H^{1 / 2}(\Gamma)$ onto $\left.H^{-1 / 2}(\Gamma)\right)$ as $\delta$ tends to 0 , or equivalently, that $\delta T_{S}(\delta, \lambda)$ converges to 0 as $\delta$ tends to 0 . To prove this result, we have to estimate the normal derivative of the function $v_{S}^{\delta} \in H^{1}\left(\Omega_{S}\right)$ 
defined by (33). The main difficulty here is that problem (33) is a singular perturbation problem when $\delta$ tends to 0 . Indeed, a detailed study of this problem (which is achieved in the appendix) shows that the normal derivative of $v_{S}^{\delta}$ on $\Gamma$ is not bounded as $\delta$ goes to 0 (this is due to the so called skin effect). More precisely, we have the following result:

Lemma 6.5. Let $v_{S}^{\delta} \in H^{1}\left(\Omega_{S}\right)$ be the solution of the boundary value problem (33) for a given trace $\varphi \in H^{1 / 2}(\Gamma)$ and a constant $\lambda>0$.

Then, for every $\nu \in] 0,1 / 2[$, there exists a constant $C>0$ (independent of $\varphi$ and $\delta$ ) such that the following estimates hold:

$$
\left\|v_{S}^{\delta}\right\|_{H^{1}} \leq C\|\varphi\|_{H^{1 / 2}(\Gamma)} \delta^{-1 / 2} \quad, \quad\left\|\triangle v_{S}^{\delta}\right\|_{L^{2}} \leq C\|\varphi\|_{H^{1 / 2}(\Gamma)} \delta^{-1+\nu} .
$$

As a consequence, we have:

$$
\left\|\partial_{n} v_{S}^{\delta}\right\|_{H^{-1 / 2}(\Gamma)} \leq C\|\varphi\|_{H^{1 / 2}(\Gamma)} \delta^{-1+\nu} .
$$

For the proof of this lemma (which is rather technical), see the appendix.

The key point now is that although the normal derivative $\partial_{n} v_{S}^{\delta}$ becomes infinite, $\delta \partial_{n} v_{S}^{\delta}$ remains bounded. This allows us to prove the following theorem, showing that the eigenvalue problems associated to $A_{\delta}$ and $A_{E}$ are close:

Theorem 6.6. Operator $T(\delta, \lambda):=\delta T_{S}(\delta, \lambda)+T_{E}(\lambda)$, where $T_{E}(\lambda)$ and $T_{S}(\delta, \lambda)$ are respectively defined by (29, 30) and (32, 33), satisfies:

$$
\forall \nu \in] 0,1 / 2\left[, \exists C>0 \text {, such that: }\left\|T(\delta, \lambda)-T_{E}(\lambda)\right\|=\left\|\delta T_{S}(\delta, \lambda)\right\| \leq C \delta^{\nu},\right.
$$

where the norm is taken in the space of bounded operators from $H^{1 / 2}(\Gamma)$ to $H^{-1 / 2}(\Gamma)$.

Proof. The result is a direct consequence of Lemma 6.5 , since for every $\varphi \in H^{1 / 2}(\Gamma)$, we have:

$$
\begin{aligned}
\left\|\left(T(\delta, \lambda)-T_{E}(\lambda)\right) \varphi\right\|_{H^{-1 / 2}} & =\delta\left\|T_{S}(\delta, \lambda) \varphi\right\|_{H^{-1 / 2}} \\
& =\delta\left\|\partial_{n} v_{S}^{\delta}\right\|_{H^{-1 / 2}(\Gamma)} \\
& \leq C\|\varphi\|_{H^{1 / 2}(\Gamma)} \delta^{\nu}
\end{aligned}
$$

where $0<\nu<1 / 2$.

Remark 6.7. The constant $C$ in Theorem 6.6 depends only on $\nu$ and $\lambda$. But as it can be seen from the proof of Lemma 6.5 detailed in the appendix, $C$ depends continuously on $\lambda$, and is thus bounded if $\lambda$ is contained in a bounded region of the complex plane.

\subsection{An asymptotic existence result for positive eigenvalues of $A$}

Using the result of Theorem 6.6 , we are going to prove that we can find in the vicinity of every positive eigenvalue of $A_{E}$, a positive eigenvalue of $A_{\delta}$, for $\delta$ small enough. This result can be stated more precisely as follows:

Theorem 6.8. Let $\left.\lambda_{E} \in\right] 0, \gamma_{\mathrm{TM}}\left[\right.$ be an eigenvalue of the operator $A_{E}$ defined by (28), and $D_{E} \subset \mathbb{C}$ a disk of center $\lambda_{E}$. We assume that the only eigenvalue of $A_{E}$ contained in $D_{E}$ is $\lambda_{E}$.

Then, for $\delta=-1 / \varepsilon_{S}$ small enough, the disk $D_{E}$ contains at least one eigenvalue $\lambda$ of $A_{\delta}=A$.

Before showing this theorem, note that this eigenvalue $\lambda$ is necessarily real (and positive if $D_{E}$ is small enough), and consequently, Theorem 6.8 gives in particular the existence result obtained in Theorem 5.6. 
Proof. We are going to use here the characterization of the eigenvalue problems associated to $A_{E}$ and $A_{\delta}$, respectively given by Propositions 6.3 and 6.4 .

So assume that $T_{E}(\xi)^{-1}$ has a pole at $\xi=\lambda_{E}$, and let us prove that there exists $\delta_{0}>0$ such that for $0<\delta<\delta_{0},(T(\delta, \xi))^{-1}=\left(\delta T_{S}(\delta, \xi)+T_{E}(\xi)\right)^{-1}$ has at least one pole in the disk $D_{E}$.

If not, there would exist a sequence $\delta_{n}$ tending to 0 , such that the family of operators $\left(T\left(\delta_{n}, \xi\right)^{-1}\right)_{n \in \mathbb{N}}$ is holomorphic on $D_{E}$. Thus, if $\mathcal{C}$ is a closed curve included in $D_{E}$ and containing $\lambda_{E}$, then:

$$
\int_{\mathcal{C}} T\left(\delta_{n}, \xi\right)^{-1} \mathrm{~d} \xi=0, \quad \forall n \in \mathbb{N} .
$$

The idea now is to take the limit $n \longrightarrow+\infty$ in this relation.

The key point of the proof is that operator $T\left(\delta_{n}, \xi\right)^{-1}$ converges to $T_{E}(\xi)^{-1}$ as $n$ tends to $+\infty$ uniformly on $\mathcal{C}$. To prove this assertion, one should first notice that:

$$
T\left(\delta_{n}, \xi\right)^{-1}-T_{E}(\xi)^{-1}=T\left(\delta_{n}, \xi\right)^{-1}\left(T_{E}(\xi)-T\left(\delta_{n}, \xi\right)\right) T_{E}(\xi)^{-1} .
$$

Now, taking for instance $\nu=1 / 4$ in Theorem 6.6, one obtains the existence of a positive constant $C$ (independent of $\xi$, see Rem. 6.7) such that:

$$
\left\|T_{E}(\xi)-T\left(\delta_{n}, \xi\right)\right\| \leq C \delta_{n}^{1 / 4}, \quad \forall \xi \in \mathcal{C}
$$

On the other hand, we have:

$$
\left\|T_{E}(\xi)^{-1}\right\| \leq C, \quad \forall \xi \in \mathcal{C} .
$$

Thus, to conclude, it remains to check that the family $\left(T\left(\delta_{n}, \xi\right)^{-1}\right)_{n \in \mathbb{N}}$ is uniformly bounded on $\mathcal{C}$. But:

$$
T\left(\delta_{n}, \xi\right)^{-1}=\left(T_{E}(\xi)+\delta_{n} T_{S}\left(\delta_{n}, \xi\right)\right)^{-1}=T_{E}(\xi)^{-1}\left(\mathrm{Id}+\delta_{n} T_{S}\left(\delta_{n}, \xi\right) T_{E}(\xi)^{-1}\right)^{-1},
$$

and by Theorem 6.6 , there exists $C>0$ such that:

$$
\left\|\delta_{n} T_{S}\left(\delta_{n}, \xi\right) T_{E}(\xi)^{-1}\right\| \leq C \delta_{n}^{1 / 4} .
$$

Thus, using the Neumann series of $\operatorname{Id}+\delta_{n} T_{S}\left(\delta_{n}, \xi\right) T_{E}(\xi)^{-1}$ one obtains the existence of $N>0$ (independent of $\xi$ ) such that for $n \geq N$ the following estimate holds:

$$
\left\|\left(\operatorname{Id}+\delta_{n} T_{S}\left(\delta_{n}, \xi\right) T_{E}(\xi)^{-1}\right)^{-1}\right\| \leq \frac{1}{1-\left\|\delta_{n} T_{S}\left(\delta_{n}, \xi\right) T_{E}(\xi)^{-1}\right\|} \leq \frac{1}{1-C \delta_{n}^{1 / 4}}, \quad \forall \xi \in \mathcal{C} .
$$

Thus, for $n \geq N$, we have:

$$
\left\|T\left(\delta_{n}, \xi\right)^{-1}\right\| \leq C, \quad \forall \xi \in \mathcal{C} .
$$

Using (37) and (38) in (36), we get:

$$
\left\|T\left(\delta_{n}, \xi\right)^{-1}-T_{E}(\xi)^{-1}\right\| \leq C \delta_{n}^{1 / 4}, \quad \forall \xi \in \mathcal{C} .
$$

One can thus take the limit in (35) and obtain:

$$
\int_{\mathcal{C}} T_{E}(\xi)^{-1} \mathrm{~d} \xi=0
$$


The contradiction comes now from the fact that since operator $T_{E}(\xi)^{-1}$ has a pole at $\xi=\lambda_{E}$, we have:

$$
\int_{\mathcal{C}} T_{E}(\xi)^{-1} \mathrm{~d} \xi=2 i \pi \operatorname{Res}\left(T_{E}(\xi)^{-1}, \xi=\lambda_{E}\right) \neq 0,
$$

where $\operatorname{Res}\left(T_{E}(\xi)^{-1}, \xi=\lambda_{E}\right)$ denotes the residue of $T_{E}(\xi)^{-1}$ at $\xi=\lambda_{E}$.

Thus, $T(\delta, \xi)^{-1}$ has necessarily a pole in $D_{E}$ for $\delta$ small enough, and Theorem 6.8 is proved.

Remark 6.9. In fact, the proof of Theorem 6.8 also shows that if the disk $D_{E}$ does not contain any eigenvalue of $A_{E}$, then it contains no eigenvalues of $A_{\delta}$. Indeed, if $T_{E}(\xi)^{-1}$ is bounded for some $\xi$ by a constant $C_{E}$, then for $\delta$ small enough, we have:

$$
\left\|T(\delta, \xi)^{-1}\right\|=\left\|T_{E}(\xi)^{-1}\left(\operatorname{Id}+\delta T_{S}(\delta, \xi) T_{E}(\xi)^{-1}\right)^{-1}\right\| \leq \frac{C_{E}}{1-C \delta^{1 / 4}} \leq C_{E}
$$

and $T(\delta, \xi)^{-1}$ is thus bounded.

\section{APPENDIX}

This appendix is devoted to the proof of Lemma 6.5, which gives an estimate of the normal derivative $\partial_{n} v_{S \mid \Gamma}^{\delta}$ of the solution $v_{S}^{\delta}$ of the boundary value problem:

$$
\left\{\begin{array}{rll}
-\delta \triangle v_{S}^{\delta}+\left(\delta \beta^{2}+\lambda\right) v_{S}^{\delta}=0 & \left(\Omega_{S}\right) \\
v_{S}^{\delta}=\varphi & (\Gamma) .
\end{array}\right.
$$

More precisely, we have to prove that for every $\nu \in] 0,1 / 2[$, there exists a constant $C>0$ (independent of $\varphi$ and $\delta$ ) such that:

$$
\begin{gathered}
\left\|v_{S}^{\delta}\right\|_{H^{1}} \leq C\|\varphi\|_{H^{1 / 2}(\Gamma)} \delta^{-1 / 2} \quad, \quad\left\|\Delta v_{S}^{\delta}\right\|_{L^{2}} \leq C\|\varphi\|_{H^{1 / 2}(\Gamma)} \delta^{-1+\nu}, \\
\left\|\partial_{n} v_{S}^{\delta}\right\|_{H^{-1 / 2}(\Gamma)} \leq C\|\varphi\|_{H^{1 / 2}(\Gamma)} \delta^{-1+\nu} .
\end{gathered}
$$

Throughout this appendix, we will denote by $\|\cdot\|_{L^{2}}$ and $\|\cdot\|_{H^{1}}$ the usual norms in $L^{2}\left(\Omega_{S}\right)$ and $H^{1}\left(\Omega_{S}\right)$, and by $(\cdot, \cdot)$ the canonical scalar product of $L^{2}\left(\Omega_{S}\right)$. Furthermore, for the sake of simplicity, $C$ will always denote a constant which is independent of $\varphi$ and $\delta$.

First, note that the last estimate follows immediately from the two first ones, since by Green's formula, we have:

$$
\left\|\partial_{n} v_{S}^{\delta}\right\|_{H^{-1 / 2}(\Gamma)} \leq C\left(\left\|\triangle v_{S}^{\delta}\right\|_{L^{2}}+\left\|v_{S}^{\delta}\right\|_{H^{1}}\right)
$$

To obtain the two first estimates, we introduce the solution $w_{S} \in H^{1}\left(\Omega_{S}\right)$ of the following elliptic boundary value problem:

$$
\left\{\begin{aligned}
-\triangle w_{S}+\beta^{2} w_{S}=0 & \left(\Omega_{S}\right) \\
w_{S}=\varphi & (\Gamma)
\end{aligned}\right.
$$

Note that $w_{S}$ satisfies

$$
\left\|w_{S}\right\|_{H^{1}}+\left\|\triangle w_{S}\right\|_{L^{2}} \leq C\|\varphi\|_{H^{1 / 2}(\Gamma)} .
$$


Then $v_{S}^{\delta}$ solves problem (33) if and only if $w_{S}^{\delta}=v_{S}^{\delta}-w_{S}$ satisfies:

$$
\left\{\begin{aligned}
-\delta \triangle w_{S}^{\delta}+\left(\delta \beta^{2}+\lambda\right) w_{S}^{\delta} & =f \quad\left(\Omega_{S}\right) \\
w_{S}^{\delta} & =0 \quad(\Gamma),
\end{aligned}\right.
$$

where:

and thus:

$$
f=-\delta \triangle w_{S}+\left(\delta \beta^{2}+\lambda\right) w_{S}=\lambda w_{S} \in H^{1}(\Omega)
$$

$$
\|f\|_{L^{2}} \leq C\|\varphi\|_{H^{1 / 2}(\Gamma)}
$$

It follows then from (40) that Lemma 6.5 will be proved if we can show that:

$$
\begin{array}{ll}
\text { (i) } & \left\|w_{S}^{\delta}\right\|_{H^{1}} \leq C\|\varphi\|_{H^{1 / 2}(\Gamma)} \delta^{-1 / 2} . \\
(i i) & \left\|\triangle w_{S}^{\delta}\right\|_{L^{2}} \leq C\|\varphi\|_{H^{1 / 2}(\Gamma)} \delta^{-1+\nu} .
\end{array}
$$

To prove $(i)$, we use the variational formulation of problem (41), which reads:

$$
a^{\delta}\left(w_{S}^{\delta}, w\right)=(f, w), \quad \forall w \in H_{0}^{1}\left(\Omega_{S}\right) .
$$

where:

$$
a^{\delta}(u, v)=\delta \int_{\Omega_{S}}\left(\nabla u \cdot \nabla v+\beta^{2} u v\right)+\lambda \int_{\Omega_{S}} u v .
$$

Choosing $w=w_{S}^{\delta}$ in (43), one gets:

$$
\left\{\begin{array}{l}
\delta\left\|\nabla w_{S}^{\delta}\right\|_{L^{2}}^{2} \leq\|f\|_{L^{2}}\left\|w_{S}^{\delta}\right\|_{L^{2}} \\
\lambda\left\|w_{S}^{\delta}\right\|_{L^{2}} \leq\|f\|_{L^{2}}
\end{array}\right.
$$

Plugging the second inequality into the first one shows that:

$$
\left\{\begin{array}{l}
\delta\left\|\nabla w_{S}^{\delta}\right\|_{L^{2}}^{2} \leq \lambda^{-1}\|f\|_{L^{2}}^{2} \\
\lambda\left\|w_{S}^{\delta}\right\|_{L^{2}} \leq\|f\|_{L^{2}}
\end{array}\right.
$$

Using (42), we obtain that:

$$
\left\{\begin{array}{l}
\delta^{1 / 2}\left\|\nabla w_{S}^{\delta}\right\|_{L^{2}} \leq C\|\varphi\|_{H^{1 / 2}} \\
\left\|w_{S}^{\delta}\right\|_{L^{2}} \leq C\|\varphi\|_{H^{1 / 2}}
\end{array}\right.
$$

and the estimate $(i)$ is thus proved .

To prove $(i i)$, we proceed as follows.

Step 1: We first notice that from (41):

$$
\triangle w_{S}^{\delta}=\delta^{-1}\left(\lambda w_{S}^{\delta}-f\right)+\beta^{2} w_{S}^{\delta}
$$


Since $\left\|w_{S}^{\delta}\right\|_{L^{2}} \leq C\|\varphi\|_{H^{1 / 2}}$, we only have to obtain an estimate on:

$$
e^{\delta}=\lambda w_{S}^{\delta}-f
$$

Step 2: Let us show that $e^{\delta}$ satisfies:

$$
\delta^{-\nu} e^{\delta} \rightarrow 0 \text { weakly in } L^{2}\left(\Omega_{S}\right), \text { for } 0<\nu<1 / 2 .
$$

Tha variational formulation (43) shows that:

$$
\left(\lambda w_{S}-f, w\right)=-\delta^{1 / 2}\left(\delta^{1 / 2} \nabla w_{S}^{\delta}, \nabla w\right)-\delta \beta^{2}\left(w_{S}^{\delta}, w\right), \quad \forall w \in H_{0}^{1}\left(\Omega_{S}\right),
$$

or equivalently:

$$
\left(\delta^{-\nu} e^{\delta}, w\right)=-\delta^{1 / 2-\nu}\left(\delta^{1 / 2} \nabla w_{S}^{\delta}, \nabla w\right)-\delta^{1-\nu} \beta^{2}\left(w_{S}^{\delta}, w\right), \quad \forall w \in H_{0}^{1}\left(\Omega_{S}\right) .
$$

Property (46) is then a direct consequence of the estimates (44).

Step 3: The previous step shows in particular that $\delta^{-\nu} e^{\delta}$ is bounded in $L^{2}\left(\Omega_{S}\right)$. Thus, the family $E^{\delta}$ of linear continuous operators defined by:

$$
E^{\delta}: \varphi \in H^{1 / 2}(\Gamma) \longrightarrow E^{\delta}(\varphi)=\delta^{-\nu} e^{\delta}=\delta^{-\nu}\left(\lambda w_{S}^{\delta}-f\right) \in L^{2}\left(\Omega_{S}\right)
$$

satisfies:

$$
\forall \varphi \in H^{1 / 2}(\Gamma), \exists C(\varphi)>0 \text { such that: }\left\|E^{\delta}(\varphi)\right\|_{L^{2}} \leq C(\varphi), \quad \forall \delta \leq 1 .
$$

The Banach-Steinhaus theorem shows then that in fact the family is uniformly bounded (with respect to $\varphi$ ). In other words:

$$
\exists C>0 \text { such that: }\left\|E^{\delta}(\varphi)\right\|_{L^{2}} \leq C\|\varphi\|_{H^{1 / 2}}, \quad \forall \delta \leq 1, \forall \varphi \in H^{1 / 2}(\Gamma) .
$$

We have thus proved that for $0<\nu<1 / 2$ :

$$
\exists C>0 \text { such that: }\left\|\lambda w_{S}^{\delta}-f\right\|_{L^{2}} \leq C\|\varphi\|_{H^{1 / 2}} \delta^{\nu}, \quad \forall \delta \leq 1, \forall \varphi \in H^{1 / 2}(\Gamma) .
$$

Summing up these results and using (45) and (44), we have proved that for every $0<\nu<1 / 2$ :

$$
\left\|\triangle w_{S}^{\delta}\right\|_{L^{2}} \leq C\|\varphi\|_{H^{1 / 2}(\Gamma)} \delta^{-1+\nu}
$$

which is nothing but $(i i)$.

\section{REFERENCES}

[1] A. Bamberger and A.-S. Bonnet, Mathematical Analysis of the Guided Modes of an Optical Fiber. SIAM J. Math. Anal. 21 (1990) 1487-1510.

[2] A.-S. Bonnet-Bendhia, G. Caloz and F. Mahé, Guided Modes of Integrated Optical Guides. A Mathematical Study. IMA J. Appl. Math. 60 (1998) 225-261.

[3] A.-S. Bonnet-Bendhia, M. Dauge and K. Ramdani, Analyse Spectrale et Singularités d'un Problème de Transmission non Coercif. C. R. Acad. Sci. Paris Sér. I 328 (1999) 717-720.

[4] A.-S. Bonnet-Bendhia, J. Duterte and P. Joly, Mathematical Analysis of Elastic Surface Waves in Topographic Waveguides. Math. Models Methods Appl. Sci. 9 (1999) 755-798. 
[5] A.-S. Bonnet-Bendhia and K. Ramdani, Mathematical Analysis of Conducting and Superconducting Transmission Lines. SIAM J. Appl. Math. 60 (2000) 2087-2113.

[6] J.-M. Cognet, Étude des modes guidés dans une ligne supraconductrice : le cas monodimensionnel. Rapport Interne $\mathrm{n}^{\circ} 295$, ENSTA, Paris (1997).

[7] R.E. Collins, Foundations for microwave engineering. Mc Graw-Hill Inc. (1992).

[8] P. Joly and C. Poirier, Mathematical Analysis of Electromagnetic Open Wave-guides. RAIRO Modèl. Math. Anal. Numér. 29 (1995) 505-575.

[9] J.G. Ma and I. Wolff, Modeling the Microwave Properties of Superconductors. IEEE Trans. Microwave Theory Tech. 43 (1995) 1053-1059.

[10] D. Marcuse, Theory of Dielectric Optical Waveguide. Academic Press, New-York (1974).

[11] K.K Mei and G. Liang, Electromagnetics of Superconductors. IEEE Trans. Microwave Theory Tech. 44 (1991) 1545-1552.

[12] A.D. Olver, Microwave and Optical Transmission. J. Wiley \& Sons Ed. (1992).

[13] K. Ramdani, Lignes Supraconductrices : Analyse Mathématique et Numérique. Ph.D. thesis, University of Paris VI, France (1999).

[14] M. Reed and B. Simon, Methods of Modern Physics, Analysis of Operators. Academic Press (1980).

To access this journal online:

www.edpsciences.org 